\title{
Localization theories for simplicial presheaves
}

\author{
P.G Goerss ${ }^{1}$ and J.F. Jardine ${ }^{2}$
}

This work was motivated in part by the following question of Soulé: given a simplicial presheaf $X$ on a site $\mathcal{C}$, how does one produce a map of simplicial presheaves $X \rightarrow L_{H \mathbb{Z}} X$ in such a way that each of the maps in sections $X(U) \rightarrow L_{H \mathbb{Z}} X(U)$, $U \in \mathcal{C}$, is an integral homology localization map in the sense of Bousfield? Secondly, if $Y$ is a simplicial presheaf which is integrally homology local in a suitable sense, is it the case that the map $X \rightarrow L_{H \mathbb{Z}} X$ induces an isomorphism

$$
\left[L_{H \mathbb{Z}} X, Y\right] \cong[X, Y]
$$

relating sets of morphisms in the homotopy category of simplicial presheaves on $\mathcal{C}$ ? These questions are related to the definition of the K-theory of simplicial sheaves that appears in [8].

The first of these questions is easily answered by observing that associated fibrations in the closed model category describing Bousfield's homology localizations are created with small object constructions and are therefore natural; in particular there is a functorial method of picking out a fibrant model $Y \rightarrow L_{H \mathbb{Z}} Y$ for arbitrary simplicial sets $Y$, which restricts in particular to a natural simplicial presheaf map $X(U) \rightarrow L_{H \mathbb{Z}} X(U), U \in \mathcal{C}$.

The second question involves homotopy coherence, and is therefore much more subtle: the analogous space-level problem can be solved by Bousfield's original techniques, but this does not imply the functorial global solution that Soule requires. The problem is solved by using methods introduced in this paper, and in particular by applying Theorem 3.9 below. In the case corresponding to the identity functor on the site $\mathcal{C}$, the chaotic topology on $\mathcal{C}$ and the constant presheaf of spectra associated to the Eilenberg-Mac Lane spectrum $H \mathbb{Z}$, Theorem 3.9 implies that there is a closed simplicial model structure in the sense of Quillen on the category $\mathbf{S}$ Pre $(\mathcal{C})$ of simplicial presheaves on $\mathcal{C}$ such that the cofibrations are the pointwise monomorphisms and the weak equivalences are the pointwise integral homology isomorphisms. The map $i: X \rightarrow L_{H \mathbb{Z}} X$ is then just a choice of fibrant model (ie. trivial cofibration, taking values in fibrant object) for this closed model structure, and the induced maps in sections $i: X(U) \rightarrow L_{H \mathbb{Z}} X(U)$ are fibrant models for the corresponding theory on simplicial sets (ie. integral homology localizations in

\footnotetext{
${ }^{1}$ Partially supported by NSF.

${ }^{2}$ Partially supported by NSERC.

This research was also supported by a NATO Collaborative Research Grant.
} 
Bousfield's original sense), because the $U$-sections functor has a left adjoint which preserves cofibrations and takes integral homology isomorphisms to pointwise integral homology isomorphisms. Furthermore, if we say that a simplicial presheaf $Y$ is integral homology local if it's fibrant with respect to this new closed model structure on $\mathbf{S} \operatorname{Pre}(\mathcal{C})$, then $Y$ is globally fibrant in the traditional sense, and the closed simplicial model structure gives an isomorphism

$$
\pi\left(L_{H \mathbb{Z}} X, Y\right) \cong \pi(X, Y)
$$

in naive homotopy classes of maps which is induced by the $H \mathbb{Z}$-trivial cofibration $i$ (here $\pi(X, Y)=\pi_{0} \operatorname{hom}(X, Y)$, for example).

This application is based on a very special case of the results that appear here, which hold in striking generality. The overall point is that a very wide class of results, which includes objects as apparently diverse as Theorem 3.9, the closed model structure for simplicial presheaves [15] (see also Remark 2.9), a general $f$ localization theory for simplicial presheaves (Theorem 4.6), the closed model structures of various stable categories (Theorem 3.7) and a homology localization technique for presheaves of spectra (Theorem 3.10), all arise from a simple collection of axioms for classes of cofibrations and weak equivalences (see axioms E1-E7 almost immediately below, and then sE1-sE7 for spectra in Section 3). The proofs, in all cases, involve relatively simple cardinality counts which are modelled simultaneously on Bousfield's original work on homology localization and the derivation of the closed model structures for simplicial presheaves. Some new theories have been discovered along the way, including a notion of localization along a geometric topos morphism (Theorem 2.7, Theorem 3.10) and a resulting method of localizing a space or a spectrum at a generalized homology theory arising from a presheaf of spectra on an arbitrary site.

There is a further application for these techniques, in that the Morel-Voevodsky $\mathbb{A}^{1}$-localization theory is an instance of the $f$-localization results of Section 4 (see Remark 4.10, but note that we do not discuss properness). The collection of known applications is, however, still quite small. It's rather difficult, in particular, to know what localization along an arbitrary geometric morphism of toposes should mean in the context of traditional homotopy theory. The fibrant objects in all of these theories continue to be really quite mysterious.

The second author would like to thank Vladimir Voevodsky for a series of conversations which helped to determine the final form of the axiom list E1-E7. 


\section{Fundamental results.}

Suppose that $\mathcal{C}$ is a small Grothendieck site, and that $\alpha$ is an infinite cardinal. A simplicial presheaf $X$ on $\mathcal{C}$ is said to be $\alpha$-bounded if $\alpha$ is an upper bound on the cardinality of all sets of sections of $X$ in the sense that $\left|X_{n}(U)\right| \leq \alpha$ for all $n \geq 0$ and all objects $U$ of $\mathcal{C}$.

Suppose that $\mathbf{E}$ is a class of morphisms of $\mathbf{S} \operatorname{Pre}(\mathcal{C})$, and say that a monomorphism of simplicial presheaves is a cofibration. Say that a simplicial presheaf cofibration which is also a member of $\mathbf{E}$ is an $\mathbf{E}$-trivial cofibration.

In many examples, we shall see that the class $\mathbf{E}$ and the class of cofibrations together satisfy the following axioms:

E1: The class of morphisms $\mathbf{E}$ is closed under retracts.

E2: Given a composable pair of morphisms

$$
X \stackrel{f}{\rightarrow} Y \stackrel{g}{\rightarrow} Z,
$$

if any two of $f, g$ and $g f$ are in the class $\mathbf{E}$, then so is the third.

E3: Every pointwise weak equivalence is in $\mathbf{E}$.

E4: The class of $\mathbf{E}$-trivial cofibrations is closed under pushout.

E5: Suppose that $\gamma$ is a limit ordinal, and there is a functor $X: \gamma \rightarrow \operatorname{SPre}(\mathcal{C})$ such that for each morphism $i \leq j$ of $\gamma$, the induced map $X(i) \rightarrow X(j)$ is an E-trivial cofibration. Then the canonical maps

$$
X(i) \stackrel{\tau_{i}}{\longrightarrow} \underset{j \in \gamma}{\lim } X(j)
$$

are E-trivial cofibrations.

E6: Suppose that the morphisms $f_{i}: X_{i} \rightarrow Y_{i}$ are $\mathbf{E}$-trivial cofibrations for $i \in I$. Then the morphism

$$
\bigsqcup_{i \in I} f_{i}: \bigsqcup_{i \in I} X_{i} \rightarrow \bigsqcup_{i \in I} Y_{i}
$$

is an $E$-trivial cofibration.

E7: There is an infinite cardinal $\alpha$ which is an upper bound for the cardinality of the set of morphisms of $\mathcal{C}$, such that for every simplicial presheaf diagram

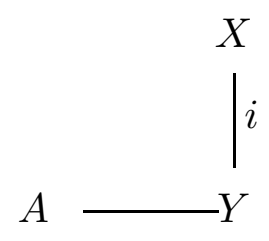


with $i$ an $E$-trivial cofibration and $A \alpha$-bounded, there is a subobject $B \subset Y$ such that $A \subset B$, the object $B$ is $\alpha$-bounded, and the inclusion $B \cap X \hookrightarrow B$ is an $E$-trivial cofibration.

We shall refer to condition $\mathbf{E} \mathbf{7}$ as the bounded cofibration condition. It is the only axiom of the list that is not a standard part of a closed model structure, and is almost always the most difficult to verify.

Say that a morphism $p: X \rightarrow Y$ is an $\mathbf{E}$-fibration if it has the right lifting property with respect to all $\mathbf{E}$-trivial cofibrations. An $\mathbf{E}$-weak equivalence is a member of the class $\mathbf{E}$.

If $K$ is a simplicial set and $X$ is a simplicial sheaf, then the tensor object $X \times K$ is defined in sections for $U \in \mathcal{C}$ by

$$
(X \times K)(U)=X(U) \times K .
$$

For the simplicial presheaves $X$ and $Y$, the function complex $\operatorname{hom}(X, Y)$ is the simplicial set whose set of $n$-simplices is defined by

$$
\operatorname{hom}(X, Y)_{n}=\operatorname{hom}\left(X \times \Delta^{n}, Y\right)
$$

where the morphism set on the right is in the category of simplicial presheaves on $\mathcal{C}$. The ordinary exponential law for simplicial sets bootstraps immediately to a simplicial category structure on the simplicial presheaf category $\mathbf{S} \operatorname{Pre}(\mathcal{C})$. The exponential object $X^{K}$ associated to a simplicial presheaf $X$ and a simplicial set $K$ is the simplicial presheaf which is defined in sections by the function spaces

$$
X^{K}(U)=\operatorname{hom}(K, X(U))
$$

for $U \in \mathcal{C}$.

TheOrem 1.1.

(1) Under the conditions $\mathbf{E 1}$ - E7 listed above, there is a closed model structure on $\mathbf{S} \operatorname{Pre}(\mathcal{C})$ such that the cofibrations are the monomorphisms, the weak equivalences are the $\mathbf{E}$-weak equivalences, and fibrations (ie. $\mathbf{E}$-fibrations) are defined by a right lifting property.

(2) Suppose further that, given an inclusion $i: K \rightarrow L$ of finite simplicial sets and a cofibration $j: X \hookrightarrow Y$, then the induced monomorphism

$$
X \times L \cup_{X \times K} Y \times K \hookrightarrow Y \times L
$$

is an $\mathbf{E}$-trivial cofibration if either $i$ is a weak equivalence of simplicial sets or $j$ is an $\mathbf{E}$-weak equivalence of simplicial presheaves. Then $\mathbf{S} \operatorname{Pre}(\mathcal{C})$ has the structure of a closed simplicial model category. 
The proof of this result is a distillation of ideas which are common to Bousfield's work on homology localizations [2], and the homotopy theory of simplicial presheaves [12].

ProOF: We only need to prove the first statement. Suppose that $\alpha$ is an infinite cardinal which is an upper bound for the cardinality of the set of morphisms of a site $\mathcal{C}$. Say that a cofibration $A \hookrightarrow B$ of $\mathbf{S} \operatorname{Pre}(\mathcal{C})$ is $\alpha$-bounded if the object $B$ is $\alpha$-bounded. We begin by showing that a morphism of $\mathbf{S} \operatorname{Pre}(\mathcal{C})$ is an $\mathbf{E}$-fibration if and only it has the right lifting property with respect to all $\alpha$-bounded cofibrations which are E-weak equivalences.

Suppose that $K$ is a simplicial set and $U$ is an object of $\mathcal{C}$, then the simplicial presheaf $L_{U} K$ is defined for $V \in \mathcal{C}$ by $L_{U} K(V)=\bigsqcup_{\phi: V \rightarrow U} K$. Observe that morphisms of simplicial presheaves $L_{U} K \rightarrow X$ are in one to one correspondence with simplicial set maps $K \rightarrow X(U)$. If the simplicial set $K$ is $\alpha$-bounded in the sense that $\left|K_{n}\right| \leq \alpha$ for $n \geq 0$, then the simplicial presheaf $L_{U} K$ is $\alpha$-bounded.

Suppose given a diagram

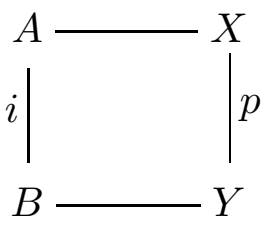

where $i$ is a cofibration and an $\mathbf{E}$-weak equivalence, and $p$ has the right lifting property with respect to all $\alpha$-bounded E-trivial cofibrations. We shall show that the indicated dotted arrow exists, making the diagram commute. Assume that the map $i$ is not an isomorphism, for otherwise the problem is solved trivially.

The object $B$ is a filtered colimit of its $\alpha$-bounded subobjects, since all generating simplicial presheaves $L_{U} \Delta^{n}$ are $\alpha$-bounded. The map $i$ is not an isomorphism, so there is an $\alpha$-bounded subobject $D$ of $B$ such $D$ is not a subobject of $A$. But then the bounded cofibration condition $\mathbf{E} 7$ says that there is an $\alpha$-bounded $E$ such that $D \subset E \subset B$, and such that the inclusion $E \cap A \hookrightarrow E$ is an E-weak equivalence. Form the diagram

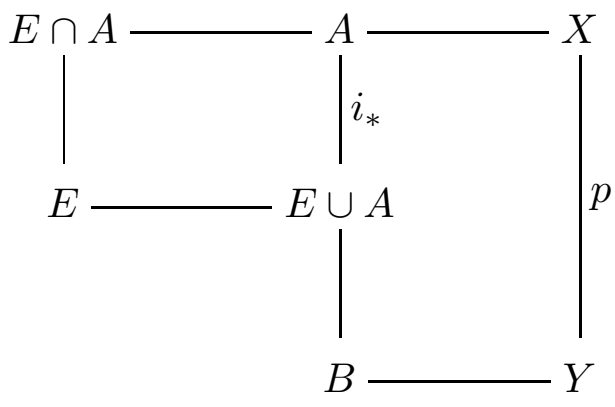


where the indicated partial lift exists since $p$ is assumed to have the right lifting property with respect to all $\alpha$-bounded cofibrations which are $\mathbf{E}$-weak equivalences. Observe further that the map $i_{*}$ is an $\mathbf{E}$-weak equivalence since the class of $\mathbf{E}$-trivial cofibrations is closed under pushout, by E4. It follows that the category of all such partial lifts is non-empty. This category has maximal elements, by a Zorn's lemma argument and E5, and any such maximal element must be a solution to the lifting problem.

Every simplicial presheaf map $f: X \rightarrow Y$ has a factorization

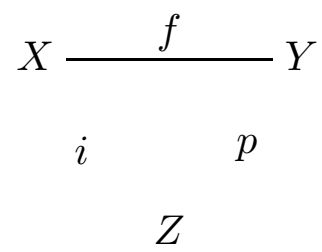

where $p$ is an $\mathbf{E}$-fibration and $i$ is a $\mathbf{E}$-trivial cofibration. In effect, take a cardinal $\beta>2^{\alpha}$. We define a functor $F: \beta \rightarrow \mathbf{S P r e}(\mathcal{C}) \downarrow Y$ by first setting $F(0)=f: X \rightarrow$ $Y$. We let

$$
X(\zeta)=\underset{\gamma<\zeta}{\lim } X(\gamma)
$$

for limit ordinals $\zeta$. Further, the map $X(\gamma) \rightarrow X(\gamma+1)$ is defined by taking the set of all diagrams

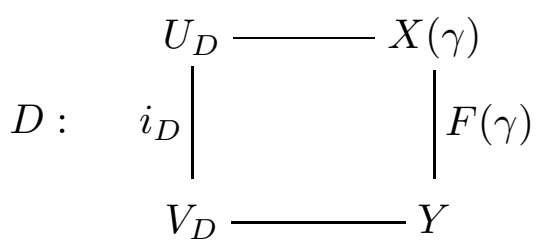

such that $i_{D}$ is an $\alpha$-bounded E-trivial cofibration, and then forming the pushout

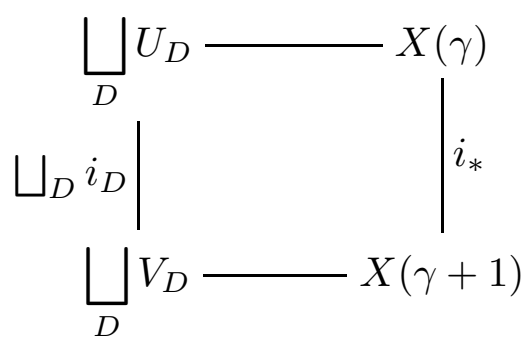


Then $i_{*}$ is an $\mathbf{E}$-trivial cofibration, by axioms $\mathbf{E} 4$ and $\mathbf{E 6}$, as is the map $i_{\beta}$ in the resulting diagram

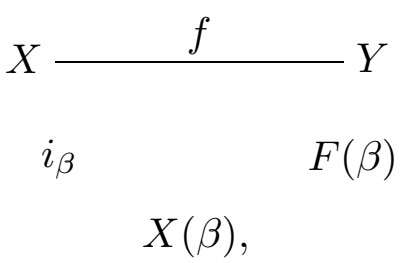

by axiom E5, where $X(\beta)=\lim _{\gamma<\beta} X(\gamma)$, and $F(\beta)$ is induced by all maps $F(\gamma)$. In any diagram

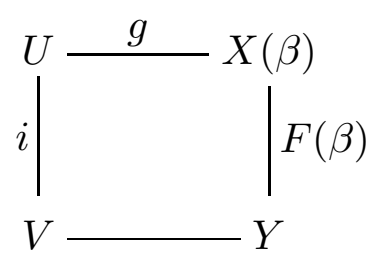

where $i$ an $\alpha$-bounded $\mathbf{E}$-trivial cofibration, the simplicial presheaf $U$ is $\alpha$-bounded, so that $g$ must factor through some subcomplex $X(\gamma) \subset X(\beta)$ with $\gamma<\beta$ (for otherwise $U$ has too many subobjects). It follows that the dotted arrow exists, making the diagram commute.

Now, if a map $f: X \rightarrow Y$ has the right lifting property with respect to all morphisms of the form $A \subset L_{U} \Delta^{n}$, then $f$ has the right lifting property with respect to all cofibrations, by an argument similar to that which characterizes $\mathbf{E}$ fibrations above. A corresponding transfinite small object argument then shows that $f$ has a factorization

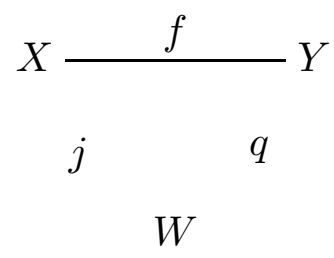

where $j$ is a cofibration, and $q$ has the right lifting property with respect to all cofibrations. In particular, $q$ is an $\mathbf{E}$-fibration; it is also a pointwise trivial fibration and hence a pointwise weak equivalence, since it has the right lifting property with respect to all maps $L_{U} \partial \Delta^{n} \subset L_{U} \Delta^{n}$. Axiom E3 says that every pointwise weak equivalence is an $\mathbf{E}$-weak equivalence, so $q$ is an $\mathbf{E}$-trivial fibration. 
We have therefore proved the factorization axioms. To verify the lifting axiom CM4, observe that if $p: X \rightarrow Y$ is a E-trivial fibration, then $p$ has a factorization

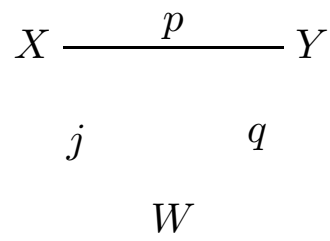

where $j$ is a cofibration and $q$ has the right lifting property with respect to all cofibrations. But then $j$ is a E-trivial cofibration by E2 and E3, since $q$ is a pointwise weak equivalence, so that there is a commutative diagram

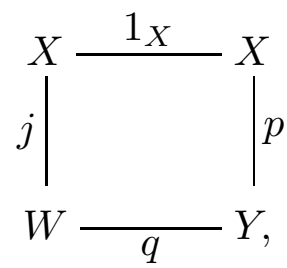

and so $p$ is a retract of $q$. In particular, $p$ has the right lifting property with respect to all cofibrations. The weak equivalence axiom CM2 is axiom E2, and the retract axiom CM3 is a direct consequence of axiom E1 and the definitions.

We say that a closed model structure on the category of simplicial presheaves on a site $\mathcal{C}$ which arises from a class $\mathbf{E}$ of cofibrations which satisfies conditions $\mathbf{E} 1$ - E7 is a localization theory. This usage is consistent with and specializes to the standard examples of localization theories in ordinary homotopy theory. There are corresponding localization theories for simplicial sheaf categories, according to the following result:

TheOrem 1.2. Suppose that the conditions E1 - E7 are satisfied for some cardinal $\alpha$ which is an upper bound on the cardinality of the set of morphisms of the site $\mathcal{C}$. Suppose further that the canonical map $\eta: X \rightarrow L^{2} X$ from a simplicial presheaf to its associated simplicial sheaf is always an $\mathbf{E}$-weak equivalence. Then we have the following:

(1) The category $\mathbf{S} S h v(\mathcal{C})$ inherits a closed model structure from the corresponding simplicial presheaf category, for which the the cofibrations are the monomorphisms, and the weak equivalences are the maps which are $\mathbf{E}$-weak equivalences of simplicial presheaves.

(2) If the simplicial presheaf category on $\mathcal{C}$ has a closed simplicial model structure with respect to these definitions, then the simplicial sheaf category $\mathbf{S} S h v(\mathcal{C})$ is also a closed simplicial model category. 
(3) The associated sheaf functor and the inclusion $\mathbf{S} S h v(\mathcal{C}) \subset \mathbf{S}$ Pre $(\mathcal{C})$ together induce an equivalence

$$
\operatorname{Ho}_{\mathbf{E}}(\mathbf{S} S h v(\mathcal{C})) \simeq \operatorname{Ho}_{\mathbf{E}}(\mathbf{S} \operatorname{Pre}(\mathcal{C}))
$$

of the respective homotopy categories.

Proof: We shall only prove statements (1) and (2). The third statement is an automatic consequence of (1) and the assumption that the canonical map $\eta: X \rightarrow$ $L^{2} X$ is an $\mathbf{E}$-weak equivalence.

To verify statement (1), observe that the closed model axioms CM1, CM2 and CM3 are immediate. The assumption on $\eta$ guarantees that the associated sheaf functor preserves $\mathbf{E}$-weak equivalences, and it follows that a map $p: X \rightarrow Y$ is an $\mathbf{E}$-fibration of simplicial sheaves if and only if $p$ is an $\mathbf{E}$-fibration of simplicial presheaves. The lifting axiom CM4 for simplicial sheaves on $\mathcal{C}$ is an easy consequence.

It therefore remains only to prove the factorization axiom CM5. We will show that any map $f: X \rightarrow Y$ of simplicial sheaves has a factorization

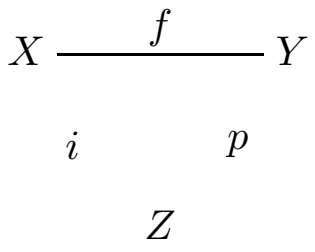

where $Z$ is a simplicial sheaf, $p$ is an $\mathbf{E}$-fibration and $i$ is a $\mathbf{E}$-trivial cofibration. The other part of CM5 has a similar proof.

As before, take a cardinal $\beta>2^{\alpha}$, and form the factorization

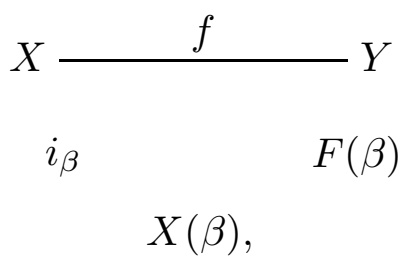

in the category of simplicial sheaves on $\mathcal{C}$, by analogy with the transfinite small object argument appearing in the proof of Theorem 1.1. In particular, $X(\beta)$ is the colimit in the simplicial sheaf category of a functor $X: \beta \rightarrow \mathbf{S} S h v(\mathcal{C})$ having

$$
X(\zeta)=\underset{\gamma<\zeta}{\lim _{\gamma}} X(\gamma)
$$


at all limit ordinals $\zeta \leq \beta$. The map $X(\gamma) \rightarrow X(\gamma+1)$ is defined by taking the set of all diagrams

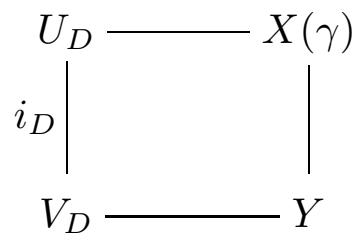

of simplicial presheaf morphisms with $i_{D}$ an $\alpha$-bounded $\mathbf{E}$-trivial cofibration, and then forming the pushout

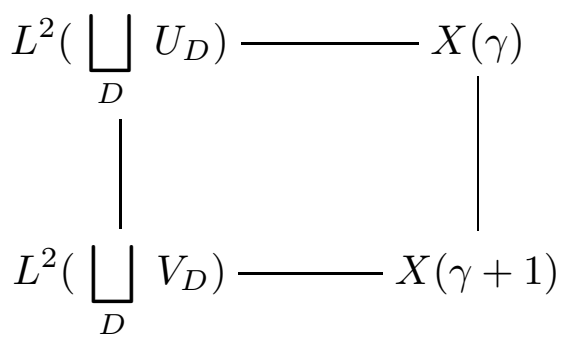

of simplicial sheaves. Then the induced canonical map $i_{\beta}: X \rightarrow X(\beta)$ is a trivial E-cofibration of simplicial sheaves.

To prove that the map $F(\beta): X(\beta) \rightarrow Y$ is an E-fibration it suffices to show that any simplicial presheaf map $g: B \rightarrow X(\beta)$ has a factorization

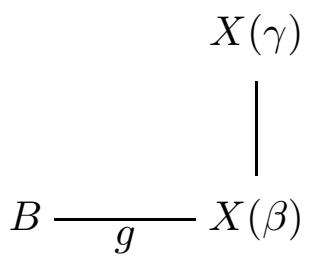

for some $\gamma<\beta$, provided that $B$ is $\alpha$-bounded.

Suppose that this is not so. Write $B(\gamma)=B \times_{X(\beta)} X(\gamma)$. Then the idea is to show that, for each $\gamma<\beta$, there is an ordinal $\zeta$ with $\gamma<\zeta<\beta$ and such that $B(\zeta)-B(\gamma)$ is non-empty. This would give a contradiction, for then the $\alpha$-bounded simplicial presheaf $B$ would have too many subobjects.

Finally, if $B(\gamma) \neq B$, then there is a section $x \in B(U)$ such that $g(x)$ is not in $X(\gamma)(U)$. The element $g(x)$ lifts to elements $y_{V}$ in the presheaf colimit $\lim _{\zeta<\beta} X(\zeta)$, 
after refinement along members $\phi: V \rightarrow U$ of a covering sieve for $U$, since the associated sheaf map

$$
\eta: \underset{\zeta<\beta}{\lim } X(\zeta) \rightarrow X(\beta)
$$

is a local epimorphism. At least one of the sections $y_{V}$ is not in $X(\gamma)(V)$, for otherwise $g(x)$ is in the image of the simplicial sheaf monomorphism $X(\gamma) \rightarrow X(\beta)$. We can therefore assume that $B$ has a section $x$ such that $g(x)$ lifts to some $z \in$ $\lim _{\zeta<\beta} X(\zeta)$ but is not in $X(\gamma)$. But then $z \in X(\zeta)$ for some $\zeta$ with $\gamma<\zeta<\beta$, so we're done.

For statement (2), suppose that $X$ is a simplicial sheaf and that $K$ is a simplicial set. The tensor object $X \otimes K$ is defined to be the simplicial sheaf $L^{2}(X \times K)$ which is obtained by applying the associated sheaf functor to the corresponding tensor object in the simplicial presheaf category. The function complex $\operatorname{hom}(X, Y)$ is the simplicial set having $n$-simplices

$$
\operatorname{hom}(X, Y)_{n}=\operatorname{hom}\left(X \otimes \Delta^{n}, Y\right)
$$

where the morphisms on the right are in the simplicial sheaf category. The simplicial category structure on $\mathbf{S} S h v(\mathcal{C})$ is then induced from the simplicial category structure on the simplicial presheaf category, through the associated sheaf functor.

Assume that $\mathbf{S P r e}(\mathcal{C})$ has the structure of a closed simplicial model category with respect to the ambient definitions. Suppose that $j: X \hookrightarrow Y$ is a cofibration of simplicial sheaves and an that $i: K \hookrightarrow L$ is an inclusion of simplicial sets. Then the induced monomorphism

$$
X \otimes L \cup_{X \otimes K} Y \otimes K \hookrightarrow Y \otimes L
$$

of simplicial sheaves is obtained from the corresponding monomorphism

$$
X \times L \cup_{X \times K} Y \times K \hookrightarrow Y \times L
$$

by applying the associated sheaf functor. If either $i$ is a weak equivalence or $j$ is an $\mathbf{E}$-weak equivalence, the simplicial presheaf map (1.4) is an $\mathbf{E}$-weak equivalence by assumption. The associated sheaf functor preserves $\mathbf{E}$-weak equivalences, so that the map (1.3) is an $\mathbf{E}$-weak equivalence.

\section{Geometric morphisms.}

We shall follow Mac Lane and Moerdijk [16] in saying that a morphism of Grothendieck sites is a functor $F: \mathcal{C} \rightarrow \mathcal{D}$ such that

(a) $\mathcal{C}$ and $\mathcal{D}$ are sites having all finite limits, and $F$ preserves them

(b) If $R \subset \operatorname{hom}(, U)$ is a covering sieve for $U \in \mathcal{C}$, then the image $F(R)$ of the collection of morphisms of $R$ under $F$ generates a covering sieve for $F(U)$. 
In the presence of a site morphism, if $X$ is a sheaf on $\mathcal{D}$, then the composite functor $X \circ F$ is a sheaf on $\mathcal{C}$ (which is denoted by $F_{*} X$ and called the direct image of $X$ along $F$ ) on account of the second condition; this condition is often paraphrased by saying that $F$ is continuous.

REMARK 2.1. Any geometric morphism $f: \mathcal{F} \rightarrow \mathcal{E}$ is induced by a site morphism $F: \mathcal{C} \rightarrow \mathcal{D}$, for a suitable choices of sites $\mathcal{C}$ and $\mathcal{D}$ underlying the toposes $\mathcal{E}$ and $\mathcal{F}$ respectively. This is a consequence of the inner workings of Giraud's Theorem. Implicitly, the claim that $\mathcal{E}$ is a Grothendieck topos means, in part, that $\mathcal{E}$ has a small set of generators $G(\mathcal{E})$. The topos $\mathcal{E}$ has all finite limits, so that the original set of generators can be expanded to a new set of generators that we shall call $\mathrm{Ob}(\mathcal{C})$ which is closed under finite limits. The full subcategory $\mathcal{C} \subset \mathcal{E}$ on this set of generators comes equipped with a canonical choice of Grothendieck topology (the covering families are the epimorphic families), in such a way that the sheaf category $\operatorname{Shv}(\mathcal{C})$ is equivalent to the topos $\mathcal{E}$. The corresponding set $\mathrm{Ob}(\mathcal{D})$ of generators for the topos $\mathcal{F}$ is obtained by closing up the set of objects $G(\mathcal{F}) \cup f^{*}(\mathrm{Ob}(\mathcal{C}))$ under finite limits. The full subcategory $\mathcal{D} \subset \mathcal{F}$ has a canonical topology such that $\operatorname{Shv}(\mathcal{D})$ is equivalent to the topos $\mathcal{F}$, and the inverse image functor $f^{*}: \mathcal{E} \rightarrow \mathcal{F}$ restricts to a site morphism $f^{\prime}: \mathcal{C} \rightarrow \mathcal{D}$ which induces the geometric morphism $f$ in the sense that $f_{*}$ can be identified up to isomorphism with composition with $f^{\prime}$, and $f^{*}$ is equivalent to $f^{\prime *}$.

Not to worry: any continous map of topological spaces or any scheme homomorphism induces a site morphism between the corresponding Grothendieck sites straight up, for all of the favourite topologies, since all such sites have finite limits which are preserved by the corresponding functors.

Recall [12], [15] that the category $\mathbf{S} \operatorname{Pre}(\mathcal{C})$ of simplicial presheaves on a small Grothendieck site is a proper closed simplicial model category in which the cofibrations are the monomorphisms. The weak equivalences of simplicial presheaves on $\mathcal{C}$ are the local weak equivalences, which can be described as maps that induce isomorphisms in all local sheaves of homotopy groups: in the case where the topos $S h v(\mathcal{C})$ has enough points, this means that a local weak equivalence is a map which induces a weak equivalence of ordinary simplicial sets in all stalks. The fibrations of $\mathbf{S} \operatorname{Pre}(\mathcal{C})$ are the global fibrations, which are those maps which have the right lifting property with respect to all maps which are simultaneously local weak equivalences and cofibrations. The associated sheaf maps $\eta: X \rightarrow L^{2} X$ are local weak equivalences, and the proper closed simplicial model structure on $\mathbf{S} \operatorname{Pre}(\mathcal{C})$ restricts to a proper closed simplicial model structure on the category $\mathbf{S} S h v(\mathcal{C})$ for which the cofibrations are the monomorphisms, the weak equivalences (respectively fibrations) are those maps of simplicial sheaves which are local weak equivalences (respectively global fibrations) of simplicial presheaves. The associated sheaf functor and the inclusion functor $\mathbf{S} S h v(\mathcal{C}) \hookrightarrow \mathbf{S} \operatorname{Pre}(\mathcal{C})$ both preserve weak equivalences, and induce 
an adjoint equivalence

$$
\operatorname{Ho}(\mathbf{S} S h v(\mathcal{C})) \simeq \operatorname{Ho}(\mathbf{S} \operatorname{Pre}(\mathcal{C}))
$$

of associated homotopy categories.

The reader who has visited the first section of this paper might now be experiencing a bit of déja vu, for good reason: the method of proof of the existence of the closed model structure on $\mathbf{S} \operatorname{Pre}(\mathcal{C})$ that appears in [15] can be interpreted as showing that the collection of local weak equivalences, suitably defined, satisfies axioms E1 - E7, so that Theorems 1.1 and 1.2 apply. In other words, the standard homotopy theory of simplicial presheaves and sheaves on an arbitrary small Grothendieck site $\mathcal{C}$ is a type of localization theory.

More explicitly, recall that a Boolean localization of the topos $S h v(\mathcal{C})$ is a geometric morphism $\wp: S h v(\mathcal{B}) \rightarrow S h v(\mathcal{C})$ such that $\mathcal{B}$ is a complete Boolean algebra and such that the associated inverse image functor $\wp^{*}: \operatorname{Shv}(\mathcal{C}) \rightarrow \operatorname{Shv}(\mathcal{B})$ is faithful. Any topos $S h v(\mathcal{C})$ has a Boolean localization - this is a result of Barr and Diaconescu [16]. The game, as it's played in [15], is first to show that the axiom of choice for the Boolean topos $\operatorname{Shv}(\mathcal{B})$ implies that every locally fibrant simplicial presheaf on $\mathcal{B}$ is actually a presheaf of Kan complexes, and that every local weak equivalence $f: X \rightarrow Y$ of simplicial sheaves on $\mathcal{B}$ is a weak equivalence of simplicial sets in each section. We've always known, one way or another (but see Lemma 2.6 below), that inverse image functors preserve local weak equivalences, so one is led to decree that a local weak equivalence on simplicial presheaves on $\mathcal{C}$ is a map $g: Z \rightarrow W$ such that the induced map $g_{*}: \wp^{*} L^{2} \mathrm{Ex}^{\infty} Z \rightarrow \wp^{*} L^{2} \mathrm{Ex}^{\infty} W$ is a local (hence pointwise) weak equivalence of sheaves of Kan complexes on $\mathcal{B}$. Note the use of Kan's $\mathrm{Ex}^{\infty}$-functor [9]; this is a functorial and combinatorial method of replacing a simplicial set by a Kan complex, and hence can be applied to simplicial presheaves.

The faithfulness of the inverse image functor $\wp^{*}$ is then used to show that this definition of local weak equivalence coincides with the standard notion involving isomorphisms of local sheaves of homotopy groups. On closer examination, one sees that the faithfulness of the functor $\wp^{*}$ only appears at the very end, and is in fact independent of the existence of a closed model structure for the simplicial presheaf category $\mathbf{S} \operatorname{Pre}(\mathcal{C})$.

This is the point of this section: we show here that any geometric morphism $f: \mathcal{E} \rightarrow \operatorname{Shv}(\mathcal{C})$ determines a closed model structure on $\mathbf{S P r e}(\mathcal{C})$ for which the cofibrations are the monomorphisms and the weak equivalences are those maps $g: X \rightarrow Y$ for which the induced maps $f^{*} L^{2} g: f^{*} L^{2} X \rightarrow f^{*} L^{2} Y$ are local weak equivalences of simplicial objects of $\mathcal{E}$. This result is Theorem 2.7. This closed model structure coincides with the standard one on $\mathbf{S} \operatorname{Pre}(\mathcal{C})$ if the inverse image functor $f^{*}: S h v(\mathcal{C}) \rightarrow \mathcal{E}$ is faithful (see Remark 2.9), but is otherwise new.

There is a technical assumption, which will appear from time to time in what follows, that $f: \mathcal{C} \rightarrow \mathcal{D}$ is a continuous functor between small sites which induces 
a geometric morphism $\operatorname{Shv}(\mathcal{D}) \rightarrow \operatorname{Shv}(\mathcal{C})$. The functor $f$ is the sort of thing that arises most easily when one wants to construct a site level functor underlying a geometric topos morphism $\mathcal{E} \rightarrow S h v(\mathcal{C})$ according to the trick involving Giraud's Theorem which is described in Remark 2.1, and in the case where one has reason to avoid adding finite limits to the site $\mathcal{C}$ inside the topos $\operatorname{Shv}(\mathcal{C})$. It is typical, for example, that one wouldn't want to fatten up the site $\mathcal{C}$ in any way when the focus of concern is the category of simplicial presheaves on $\mathcal{C}$. Note that there is a fairly extensive discussion in [16] concerning the question of when a functor $\mathcal{C} \rightarrow \mathcal{D}$ between Grothendieck sites induces a geometric morphism; in their terminology a continuous functor $f: \mathcal{C} \rightarrow \mathcal{D}$ induces a geometric morphism $\operatorname{Shv}(\mathcal{D}) \rightarrow \operatorname{Shv}(\mathcal{C})$ if and only if $f$ is also "flat", or equivalently "filtering".

We begin by establishing a technical condition (Lemma 2.3) which leads to instances of the bounded cofibration axiom E7 that hold quite generally for base change along any functor between Grothendieck sites.

Suppose that $f: X \rightarrow Y$ is a morphism of simplicial presheaves on a small Grothendieck site $\mathcal{C}$, and let $i: K \subset L$ be an inclusion of finite simplicial sets. We say that $f$ has the local right lifting property with respect to $i$ if given any commutative diagram of simplicial set maps

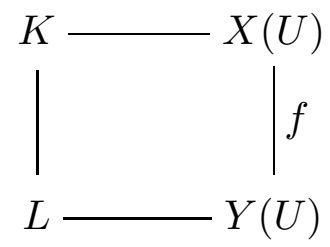

and for any object $U$ of $\mathcal{C}$ there is a covering sieve $R \subset \operatorname{hom}(, U)$ such that for each $\phi: V \rightarrow U$ in $R$ there is a commutative diagram of simplicial set maps

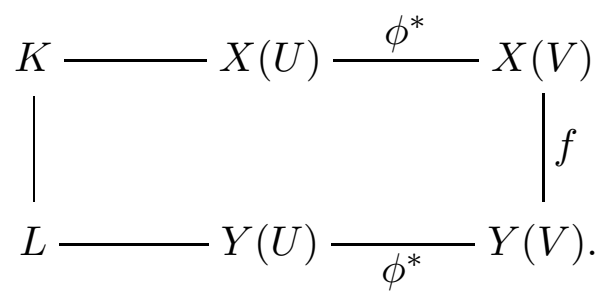

In other words, all lifting problems having the form of diagram (2.2) have solutions after refinement along covering sieves. This condition is equivalent, variously, to the requirement that the induced sheaf map

$$
L^{2} X^{L} \stackrel{\left(i^{*}, f_{*}\right)}{\longrightarrow} L^{2} X^{K} \times_{L^{2} Y^{K}} L^{2} Y^{L}
$$


is a sheaf epimorphism, and to the requirement that the presheaf map

$$
X^{L} \stackrel{\left(i^{*}, f_{*}\right)}{\longrightarrow} X^{K} \times_{Y^{K}} Y^{L}
$$

is a local epimorphism in the sense that all sections of the base lift to $X^{L}$ after refinement along some covering sieve. Here, for example, $X^{L}$ is the presheaf on $\mathcal{C}$ which is defined via simplicial set maps in sections by the formula

$$
X^{L}(U)=\operatorname{hom}_{\mathbf{S}}(L, X(U)) .
$$

Suppose that the functor $F: \mathcal{C} \rightarrow \mathcal{D}$ is any functor between two Grothendieck sites. The direct image functor $F_{*}: \operatorname{Pre}(\mathcal{D}) \rightarrow \operatorname{Pre}(\mathcal{C})$ is nevertheless defined by composition with $F$ - it's just that $F_{*}$ might not preserve sheaves. Furthermore, the functor $F_{*}$ always has a left adjoint $F^{p}: \operatorname{Pre}(\mathcal{C}) \rightarrow \operatorname{Pre}(\mathcal{D})$, which can be defined explicitly for a presheaf $Y$ on $\mathcal{D}$, in sections, by

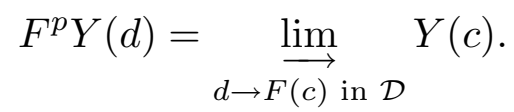

If the cardinal $\alpha$ is an upper bound for the size of the set of morphisms of $\mathcal{D}$ and the presheaf $Y$ on $\mathcal{C}$ is $\alpha$-bounded, then the presheaf $F^{p} Y$ is $\alpha$-bounded.

Lemma 2.3. Suppose that $F: \mathcal{C} \rightarrow \mathcal{D}$ is any functor between small Grothendieck sites. Let $\alpha$ be an infinite cardinal which is an upper bound on the cardinalities of the sets of morphisms for the categories $\mathcal{C}$ and $\mathcal{D}$. Suppose that $f: X \rightarrow Y$ is a map of simplicial presheaves on $\mathcal{C}$ and that $Z \subset Y$ is an $\alpha$-bounded subcomplex of $Y$. Assume further that

(1) $F^{p} f: F^{p} X \rightarrow F^{p} Y$ has the local right lifting property with respect to a family $K_{i} \subset L_{i}$ of simplicial set monomorphisms, where $L_{i}$ is finite, $i \in I$, and $|I|<\alpha$,

(2) there is a functor defined on the $\alpha$-bounded subcomplexes $W \subset Y$ with $Z \subset W$, taking values in commutative diagrams of simplicial presheaf maps of the form

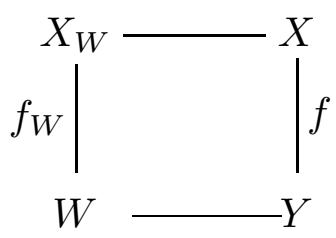

such that $X_{W}$ is $\alpha$-bounded, and such that $f=\lim _{Z \subset W} f_{W}$ and further that the functor $V \mapsto f_{V}$ preserves filtered colimits in the category of $\alpha$-bounded subobjects $V \subset X$ with $Z \subset V$. 
Then there is some $\alpha$-bounded subcomplex $W \subset Y$ with $Z \subset W$ such that the map $F^{p} f_{W}: F^{p} X_{W} \rightarrow F^{p} W$ has the local right lifting property with respect to all $K_{i} \subset L_{i}$.

Proof: The functor $F^{p}$ has a right adjoint, so that $V \mapsto F^{p} f_{V}$ preserves filtered colimits in $V$.

The simplicial presheaves $F^{p} X_{Z}$ and $F^{p} Z$ are $\alpha$-bounded, so there is an $\alpha$ bounded collection of diagrams of simplicial set maps

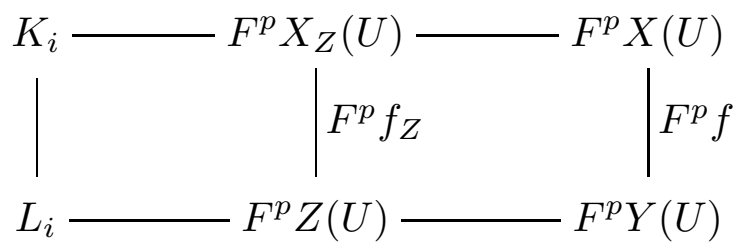

Given such a diagram, there is a covering sieve $R \subset \operatorname{hom}(U$,$) such that for all$ $\phi: V \rightarrow U$ there is a lifting

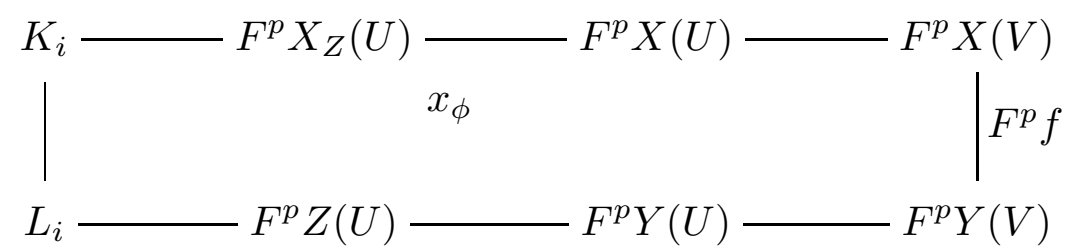

The simplicial set $L_{i}$ is finite, and $F^{p} X=\lim _{Z \subset W} F^{p} X_{W}$. It follows that there is an $\alpha$-bounded $W^{\prime}$ with $Z \subset W^{\prime}$ such that all $x_{\phi}$ live in $F^{p} X_{W^{\prime}}$. Taking the union of all such subcomplexes $W^{\prime}$ over all diagrams (2.4) gives an $\alpha$-bounded subcomplex $Z_{1}$ of $Y$ such that $Z \subset Z_{1}$ and such that all lifting problems (2.4) are solved in $F^{p} X_{Z_{1}}$. Repeat the construction of obtain a sequence of $\alpha$-bounded subobjects

$$
Z=Z_{0} \subset Z_{1} \subset Z_{2} \subset \ldots
$$

such that all local lifting problems

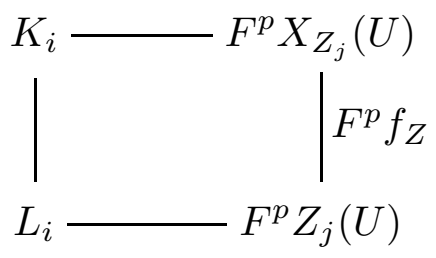


are solved over $Z_{j+1}$.

Let $W$ be the $\alpha$-bounded subcomplex defined by

$$
W=\cup_{j \geq 0} Z_{j}
$$

Then $F^{p} X_{W}=\underline{\lim }_{j \geq 0} F^{p} X_{Z_{j}}$, and any map $K_{i} \rightarrow F^{p} X_{W}(U)$ factors through some map $K_{i} \rightarrow F^{p} \bar{X}_{Z_{j}}(U)$ since $K_{i}$ is finite. It follows that that the induced map $F^{p} f_{W}: F^{p} X_{W} \rightarrow F^{p} W$ has the local right lifting property with respect to all $K_{i} \subset L_{i}$.

REMARK 2.5. There is a natural source of functors $V \mapsto f_{V}$ satisfying condition (2) of Lemma 2.3. Suppose that $f: X \rightarrow Y$ is a map of simplicial presheaves on a site $\mathcal{C}$. Then $f$ has a factorization

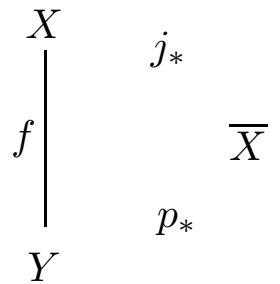

such that $p_{*}$ is a pointwise Kan fibration and $j_{*}$ is a pointwise weak equivalence, and this factorization is natural and preserves filtered colimits in $f$. In effect, take the standard factorization

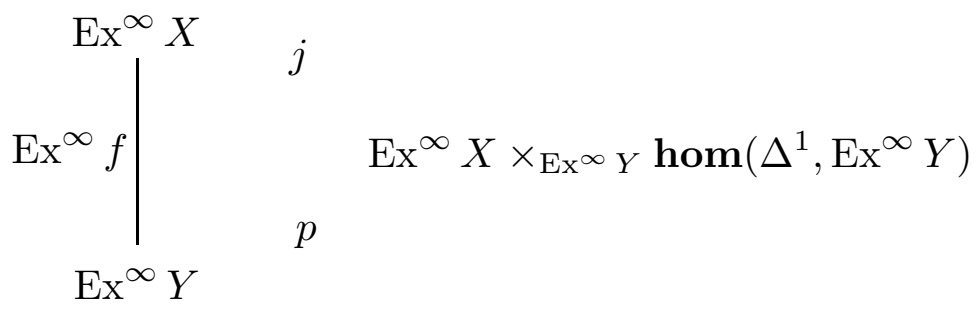

and pull it back to $Y$ using the diagram

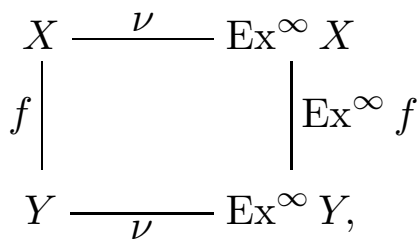


so that

$$
\bar{X}=Y \times_{\mathrm{Ex}^{\infty} Y} \operatorname{Ex}^{\infty} X \times_{\operatorname{Ex}^{\infty} Y} \operatorname{hom}\left(\Delta^{1}, \operatorname{Ex}^{\infty} Y\right)
$$

The maps $\nu$ are pointwise weak equivalences, and weak equivalences are preserved by pullback along fibrations in the simplicial set category, so the induced map $j_{*}$ is a pointwise weak equivalence. This construction is natural in morphisms $f$, and preserves filtered colimits in the category of simplicial presheaf morphisms. Note finally that if $X$ and $Y$ are $\alpha$-bounded simplicial presheaves, then so is $\bar{X}$.

It follows, for example, that if $g: X \rightarrow Y$ is a morphism of simplicial presheaves such that $g^{-1}(W)$ is $\alpha$-bounded whenever $W$ is an $\alpha$-bounded subcomplex of $Y$, then the functor defined on $\alpha$-bounded subcomplexes $W$ of $Y$ containing a fixed $\alpha$-bounded subobject $Z$ by sending $W$ to the pointwise fibration $p_{*}: \overline{g^{-1}(W)} \rightarrow W$ converges to the map $p_{*}: \bar{X} \rightarrow Y$, and thus satisfies condition (2) of Lemma 2.3, for $f=p_{*}$.

Lemma 2.6. Suppose that $f: \mathcal{C} \rightarrow \mathcal{D}$ is a continuous functor between small Grothendieck sites which induces a geometric morphism $\operatorname{Shv}(\mathcal{D}) \rightarrow \operatorname{Shv}(\mathcal{C})$. Then

$(1)$ The functors $f^{p}: \mathbf{S} \operatorname{Pre}(\mathcal{C}) \rightarrow \mathbf{S P r e}(\mathcal{D})$ and $f^{*}: \mathbf{S} \operatorname{Shv}(\mathcal{C}) \rightarrow \mathbf{S} \operatorname{Shv}(\mathcal{D})$ preserve local weak equivalences.

(2) If $p: X \rightarrow Y$ is a global fibration of simplicial sheaves on $\mathcal{D}$, then the direct image $f_{*} p: f_{*} p: f_{*} X \rightarrow f_{*} Y$ is a global fibration of $\mathbf{S} S h v(\mathcal{C})$. If the functor $f$ preserves finite limits, then $f_{*}$ preserves global fibrations of simplicial presheaves.

ProOF: Only the statement about preservation of local weak equivalences on the simplicial presheaf level requires proof, because the functor $f^{*}$ preserves cofibrations in general, and $f^{p}$ preserves cofibrations under the extra exactness condition of $f$.

Suppose that $g: X \rightarrow Y$ is a local weak equivalence of $\operatorname{SPre}(\mathcal{C})$. We want to show that $f^{*} L^{2} g \cong L^{2} f^{p} g$ is a local weak equivalence of simplicial presheaves over $\mathcal{D}$, for then $f^{p} g$ is also a local weak equivalence.

The functor $f^{*} L^{2}$ preserves the $\mathrm{Ex}^{\infty}$ construction: in effect, $f^{*} L^{2} \mathrm{Ex}^{\infty} X \cong$ $L^{2} \mathrm{Ex}^{\infty} f^{*} L^{2} X$ by the exactness of $f^{*}$, and the associated sheaf functor $L^{2}$ takes pointwise weak equivalences to local weak equivalences. It follows that $f^{*} L^{2} g$ is a local weak equivalence over $\mathcal{D}$ if and only if $f^{*} L^{2} \mathrm{Ex}^{\infty} g$ is a local weak equivalence. It therefore suffices to assume that $g: X \rightarrow Y$ is a morphism of presheaves of Kan complexes. Then there is a commutative diagram

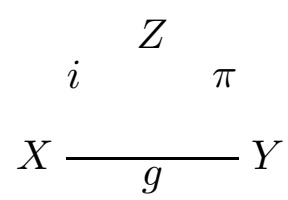


in which $\pi$ is a pointwise Kan fibration and $i$ is right inverse to a pointwise trivial fibration $\pi^{\prime}: Z \rightarrow X$. But then $\pi$ is a local weak equivalence and a pointwise Kan fibration between presheaves of Kan complexes, and therefore has the local right lifting property with respect to all inclusions $\partial \Delta^{n} \subset \Delta^{n}$, as does the map $\pi^{\prime}$. The functor $f^{*} L^{2}$ preserves this local right lifting property, by exactness, so that the maps $f^{*} L^{2} \pi$ and $f^{*} L^{2} \pi^{\prime}$ are local weak equivalences over $\mathcal{D}$, as is $f^{*} L^{2} g$.

Suppose that $f: \mathcal{E} \rightarrow S h v(\mathcal{C})$ is a geometric topos morphism, and consider the composite functor

$$
\mathbf{S} \operatorname{Pre}(\mathcal{C}) \stackrel{L^{2}}{\longrightarrow} \mathbf{S} \operatorname{Sh} v(\mathcal{C}) \stackrel{f^{*}}{\longrightarrow} \mathbf{S} \mathcal{E} .
$$

Then we know that the category of simplicial objects in $\mathcal{E}$ is a proper closed simplicial model category in a canonical way. Say that a map $g: Z \rightarrow W$ of simplicial presheaves on $\mathcal{C}$ is an $f^{*} L^{2}$-weak equivalence if the induced map $f^{*} L^{2}(g): f^{*} L^{2} Z \rightarrow f^{*} L^{2} W$ is a local weak equivalence of the category $\mathbf{S} \mathcal{E}$ of simplicial objects in the Grothendieck topos $\mathcal{E}$.

Theorem 2.7. Suppose that $f: \mathcal{E} \rightarrow \operatorname{Shv}(\mathcal{C})$ is a geometric morphism, where $\mathcal{C}$ is a small site. Then the composite functor $f^{*} L^{2}: \mathbf{S} \operatorname{Pre}(\mathcal{C}) \rightarrow \mathbf{S E}$ induces a proper closed simplicial model category structure on $\mathbf{S} \operatorname{Pre}(\mathcal{C})$, for which the $\left(f^{*}\right.$-local) weak equivalences are the $f^{*} L^{2}$-weak equivalences, the cofibrations are inclusions, and the ( $f^{*}$-global) fibrations are defined by a right lifting property.

Proof: This result is proved by appealing to Theorem 1.1, where the morphism class $\mathbf{E}$ is the collection of $f^{*} L^{2}$-weak equivalences. We can assume that $f$ is induced by a continuous functor $f: \mathcal{C} \rightarrow \mathcal{D}$. We know from Lemma 2.6 that the functor $f^{*} L^{2}$ preserves local weak equivalences, and therefore takes pointwise weak equivalences to local weak equivalences, giving E3.

The class of local trivial cofibrations of $\mathbf{S} S h v(\mathcal{D})$ is closed under pushout in the standard closed model structure for the closed model structure on $\mathcal{D}$, and the functor $f^{*} L^{2}$ is left and right exact, so axiom E4 is satisfied. Similar exactness assertions and the closed model structure for $\mathbf{S} S h v(\mathcal{D})$ together lead to axioms E5 and E6, while $\mathbf{E} 1$ and $\mathbf{E 2}$ are trivial. It remains only to verify the bounded cofibration property E7.

Let $\alpha$ be an infinite cardinal which is an upper bound for the cardinalities of the sets of morphisms for the categories $\mathcal{C}$ and $\mathcal{D}$, and suppose given a simplicial presheaf diagram

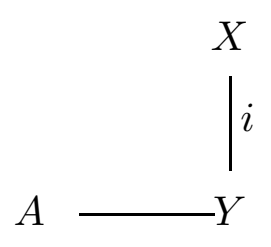


on $\mathcal{C}$, where $A$ is an $\alpha$-bounded subcomplex of $Y$ and the map $i$ is a cofibration and a $p^{*} L^{2}$-weak equivalence. As seen previously, we can apply the construction of Remark 2.5 to the map $i$ and all of the inclusions $V \cap X \hookrightarrow V$ arising from $\alpha$-bounded subcomplexes $V$ of $Y$ which contain $A$, to obtain the pointwise Kan fibration $p_{*}$ : $\bar{X} \rightarrow Y$ as a filtered colimit of the pointwise fibrations $p_{*}: \overline{V \cap X} \rightarrow V$, where the functor $V \mapsto \overline{V \cap X}$ preserves filtered colimits, and all objects $\overline{V \cap X}$ are $\alpha$ bounded. The image $L^{2} f^{p} p_{*}=f^{*} L^{2} p_{*}$ in $\mathbf{S} S h v(\mathcal{D})$ of the map $p_{*}: \bar{X} \rightarrow Y$ has the local right lifting property with respect to all inclusions $\partial \Delta^{n} \subset \Delta^{n}$. The associated sheaf functor reflects this local left lifting property, so the simplicial presheaf map $f^{p} p_{*}$ also has the local right lifting property with respect of all inclusions $\partial \Delta^{n} \subset \Delta^{n}$.

Now apply Lemma 2.3 in the case where the site-level functor is $f: \mathcal{C} \rightarrow \mathcal{D}$, the simplicial set inclusions are the morphisms $\partial \Delta^{n} \subset \Delta^{n}, n \geq 0$, the morphism $f$ is the map $p_{*}: \bar{X} \rightarrow Y$, and the functor $W \mapsto f_{W}$ in that statement is identified with the functor which takes $W$ to the pointwise fibration $p_{*}: \overline{W \cap X} \rightarrow W$. This gives and $\alpha$-bounded subcomplex $B \subset Y$ with $A \subset B$ such that the map $f^{p} p_{*}: f^{p} \overline{B \cap X} \rightarrow f^{p} B$ has the local right lifting property with respect to all inclusions $\partial \Delta^{n} \subset \Delta^{n}$. Applying the associated sheaf functor therefore shows that the map $f^{*} L^{2} p_{*}: f^{*} L^{2} \overline{B \cap X} \rightarrow f^{*} L^{2} B$ has the same local left lifting property, and is therefore a local weak equivalence of the simplicial sheaf category on $\mathcal{D}$. The corresponding inclusion $B \cap X \hookrightarrow B$ is thus part of a commutative diagram

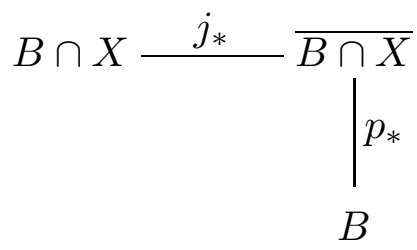

in which the maps $j_{*}$ and $p_{*}$ are $f^{*} L^{2}$-weak equivalences.

Suppose that the diagram

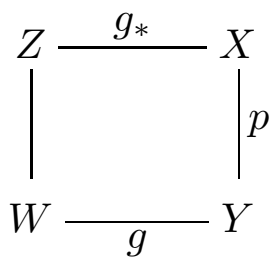

is a pullback diagram in $\operatorname{SPre}(\mathcal{C})$, where $g$ is an $f^{*} L^{2}$-weak equivalence and $p$ is an $f^{*}$-global fibration. Every local weak equivalence is an $f^{*} L^{2}$-weak equivalence, by Lemma 2.6, so that every $f^{*}$-global fibration is a global fibration, and hence a 
local fibration. It follows that applying the functor $f^{*} L^{2}$ gives a pullback diagram

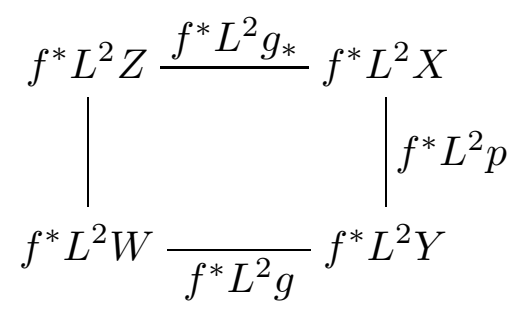

of simplicial sheaves on $\mathcal{D}$ in which $f^{*} L^{2} p$ is a local fibration and $f^{*} L^{2} g$ is a local weak equivalence. A Boolean localization argument (see the proof of Theorem 24 in [15]) shows that local weak equivalences are stable under pullback along local fibrations, so $f^{*} L^{2} g_{*}$ is a local weak equivalence.

Theorem 2.8. Suppose that $f: \mathcal{E} \rightarrow \operatorname{Shv}(\mathcal{C})$ is a geometric morphism, where $\mathcal{C}$ is a small site. Then there is a proper closed simplicial model structure on the simplicial sheaf category $\mathbf{S} S h v(\mathcal{C})$ such that the cofibrations are the monomorphisms, and a map $g: X \rightarrow Y$ is a weak equivalence if and only if the map $f^{*} g: f^{*} X \rightarrow f^{*} Y$ is a weak equivalence of $\mathbf{S E}$. Furthermore, the associated sheaf functor preserves $f^{*}$-local weak equivalences, so there is an induced adjoint equivalence

$$
\operatorname{Ho}_{f}(\operatorname{SPre}(\mathcal{C})) \simeq \operatorname{Ho}_{f}(\mathbf{S S h v}(\mathcal{C})) .
$$

Proof: Observe that the weak equivalences of simplicial sheaves on $\mathcal{C}$ are exactly those maps which are $f^{*} L^{2}$-weak equivalences in the simplicial presheaf category, and that the fibrations are $f^{*}$-global fibrations. This result is a consequence of Theorem 2.7 and Theorem 1.2. One uses Lemma 2.6 to show that that the associated sheaf map $\eta: X \rightarrow L^{2} X$ is an $f^{*} L^{2}$-weak equivalence.

REMARK 2.9. Generally, for any geometric morphism $f: \mathcal{E} \rightarrow \operatorname{Shv}(\mathcal{C})$, the functor $f^{*} L^{2}: \mathbf{S} \operatorname{Pre}(\mathcal{C}) \rightarrow \mathbf{S} \mathcal{E}$ takes local weak equivalences to local weak equivalences, by Lemma 2.6. However, if the inverse image map $f^{*}: S h v(\mathcal{C}) \rightarrow \mathcal{E}$ is also faithful, then a simplicial presheaf map $g: X \rightarrow Y$ on $\mathcal{C}$ is a local weak equivalence if and only if $f^{*} L^{2} g$ is a local weak equivalence of simplicial sheaves, so that the local weak equivalences and the $f^{*}$-local weak equivalences of $\mathbf{S} \operatorname{Pre}(\mathcal{C})$ coincide. To see this, take a map $g: X \rightarrow Y$ and form the factorization

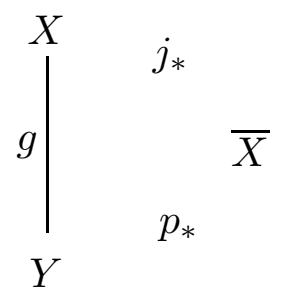


such that $p_{*}$ is a pointwise Kan fibration and $j_{*}$ is a pointwise weak equivalence, according to the method outlined in Remark 2.5. Then the composite functor $f^{*} L^{2}$ preserves local weak equivalences and local fibrations, so that $g$ is an $f^{*} L^{2}$ weak equivalence if and only if the map $f^{*} L^{2} p_{*}$ has the local right lifting property with respect to all inclusions $\partial \Delta^{n} \subset \Delta^{n}$. In the case that $f^{*}$ is faithful, the composite $f^{*} L^{2}$ reflects this local left lifting property, so therefore $g$ is an $f^{*} L^{2}$ weak equivalence if and only if the map $p_{*}$ has the local right lifting property with respect to all inclusions $\partial \Delta^{n} \subset \Delta^{n}$, and hence if and only if $g$ is a local weak equivalence.

\section{Homology theories.}

Going further requires that we first back up, and see that the conditions of Theorem 1.1 and its proof can, after minor adjustments, be used as a template for results about presheaves of spectra.

Suppose that $\mathbf{s E}$ is a class of morphisms of the category $\mathbf{S p t} \operatorname{Pre}(\mathcal{C})$ of presheaves of spectra on a site $\mathcal{C}$. A cofibration $i: X \rightarrow Y$ presheaves of spectra is the standard thing [5], [14], namely a map such that the level 0 map $i^{0}: X^{0} \rightarrow Y^{0}$ is a cofibration of pointed simplicial presheaves, as are all induced maps

$$
S^{1} \wedge Y^{n} \cup_{S^{1} \wedge X^{n}} X^{n+1} \rightarrow Y^{n+1}
$$

Say that a cofibration of presheaves of spectra which is also a member of $\mathbf{s E}$ is an sE-trivial cofibration.

For any infinite cardinal $\alpha$, a presheaf of spectra $A$ is said to be $\alpha$-bounded if each of its constituent simplicial presheaves $A^{n}, n \geq 0$, is $\alpha$-bounded. If $\alpha$ is an upper bound for the cardinality of the set of morphisms of the underlying site $\mathcal{C}$, then every presheaf of spectra $X$ is a filtered colimit of its $\alpha$-bounded subobjects. In effect, take some section $x \in X_{m}^{n}(U)$, and form the corresponding pointed simplicial presheaf map $x:\left(L_{U} \Delta^{m}\right)_{+} \rightarrow X^{n}$. The simplicial presheaf $\left(L_{U} \Delta^{m}\right)_{+}$is $\alpha$-bounded by the assumption on the size of the cardinal $\alpha$. Then $x$ canonically determines a map of presheaves of spectra $x_{*}: \Sigma^{\infty}\left(L_{U} \Delta^{m}\right)_{+}[-n] \rightarrow X$, whose image is an $\alpha$-bounded subobject of $X$ which contains the section $x$. Here,

$$
\Sigma^{\infty}\left(L_{U} \Delta^{m}\right)_{+}[-n]^{k}= \begin{cases}* & \text { if } k-n<0, \text { and } \\ \Sigma^{\infty}\left(L_{U} \Delta^{m}\right)_{+}^{k-n} & \text { if } k-n \geq 0\end{cases}
$$

according to the usual indexing conventions, and $\Sigma^{\infty}\left(L_{U} \Delta^{m}\right)_{+}$is the standard suspension spectrum object for the pointed simplicial presheaf $\left(L_{U} \Delta^{m}\right)_{+}$.

The analogue of the list of conditions E1 - E7 for Theorem 1.1 is the following collection of statements:

sE1: The class of morphisms $\mathbf{s E}$ is closed under retracts. 
sE2: Given a composable pair of morphisms

$$
X \stackrel{f}{\rightarrow} Y \stackrel{g}{\rightarrow} Z,
$$

if any two of $f, g$ and $g f$ are in the class $\mathbf{~} \mathbf{E}$, then so is the third.

sE3: Every pointwise strict equivalence is in $\mathbf{s E}$.

sE4: The class of $\mathbf{s E - t r i v i a l ~ c o f i b r a t i o n s ~ i s ~ c l o s e d ~ u n d e r ~ p u s h o u t . ~}$

sE5: Suppose that $\gamma$ is a limit ordinal, and there is a functor $X: \gamma \rightarrow \operatorname{Spt} \operatorname{Pre}(\mathcal{C})$ such that for each morphism $i \leq j$ of $\gamma$, the induced map $X(i) \rightarrow X(j)$ is an sE-trivial cofibration. Then the canonical maps

$$
X(i) \stackrel{\tau_{i}}{\longrightarrow} \underset{j \in \gamma}{\lim } X(j)
$$

are $\mathbf{s E - t r i v i a l ~ c o f i b r a t i o n s . ~}$

sE6: Suppose that the morphisms $f_{i}: X_{i} \rightarrow Y_{i}$ are $\mathbf{s E - t r i v i a l ~ c o f i b r a t i o n s ~ f o r ~}$ $i \in I$. Then the morphism

$$
\bigvee_{i \in I} f_{i}: \bigvee_{i \in I} X_{i} \rightarrow \bigvee_{i \in I} Y_{i}
$$

is an $E$-trivial cofibration.

sE7: There is an infinite cardinal $\alpha$ which is at least as large as the cardinality of the set of morphisms of $\mathcal{C}$, such that for every diagram

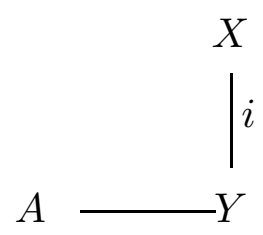

of maps of presheaves of spectra with $i$ a $\mathbf{s E - t r i v i a l ~ c o f i b r a t i o n , ~ a n d ~} A \alpha$ bounded, there is an $\alpha$-bounded subobject $B \subset Y$ such that $A \subset B$, and such that the inclusion $B \cap X \hookrightarrow B$ is an $\mathbf{s E - t r i v i a l ~ c o f i b r a t i o n . ~}$

A pointwise strict equivalence is a map $f: X \rightarrow Y$ of presheaves of spectra such that all induced maps of simplicial sets $f: X^{n}(U) \rightarrow Y^{n}(U), U \in \mathcal{C}$ are weak equivalences. Also, perhaps it's hard to believe, but the following requires proof so that the statement $\mathbf{s E 7}$ makes sense: 
Lemma 3.1. Suppose that $i: A \hookrightarrow B$ is a cofibration of spectra, and that $j: V \subset B$ is a subcomplex of $B$. Then the induced map $i_{*}: V \cap A \rightarrow V$ is a cofibration of spectra.

REMARK 3.2. When we say that $j: V \subset B$ is a subobject or subcomplex of $B$, we mean simply that all maps $j: V^{n} \rightarrow B^{n}$ are monomorphisms. We do not mean that $j$ is a cofibration.

Proof of Lemma 3.1: We need to show that all induced maps

$$
\left(S^{1} \wedge V^{n}\right) \cup_{S^{1} \wedge\left(V^{n} \cap A^{n}\right)}\left(V^{n+1} \cap A^{n+1}\right) \stackrel{\left(\sigma, i_{*}\right)}{\longrightarrow} V^{n+1}
$$

are cofibrations in the pointed simplicial set category. Given that the diagram of spectrum maps

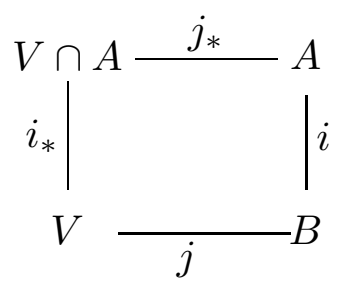

induces a commutative diagram

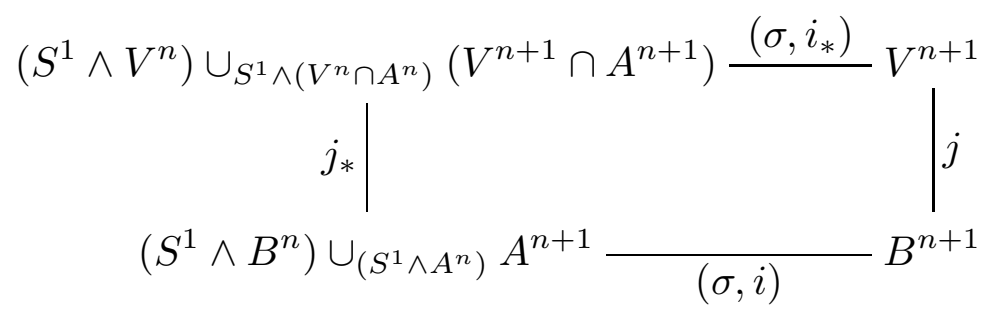

it suffices to show that the vertical map $j_{*}$ in (3.3) is a cofibration.

Note that any pointed map

$$
\left(S^{1} \wedge B^{n}\right) \cup_{\left(S^{1} \wedge A^{n}\right)} A^{n+1} \rightarrow Y
$$

can be identified with a pair of maps $f: A^{n+1} \rightarrow Y$ and $g: B^{n} \rightarrow \Omega Y$, such that the diagram

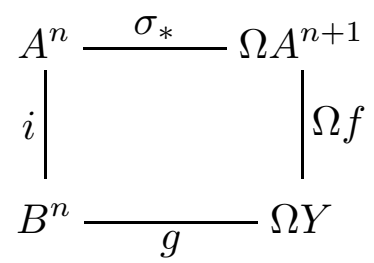


commutes, where $\sigma_{*}$ is the adjoint of the bonding map $\sigma: S^{1} \wedge A^{n} \rightarrow A^{n+1}$. In this language, suppose given a commutative diagram

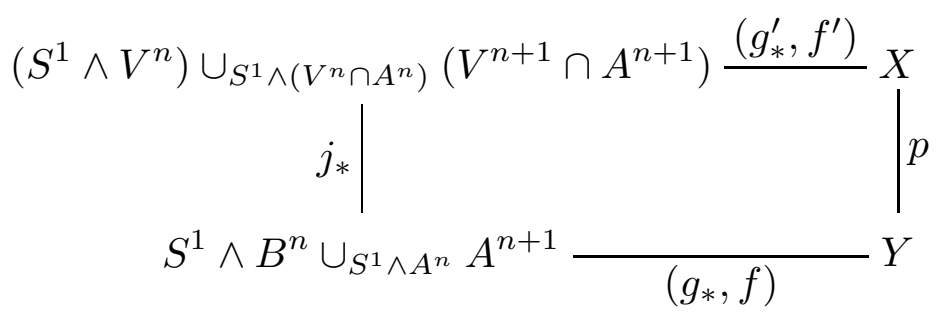

where $p$ is a trivial fibration. Then there is a map $h$ making the diagram

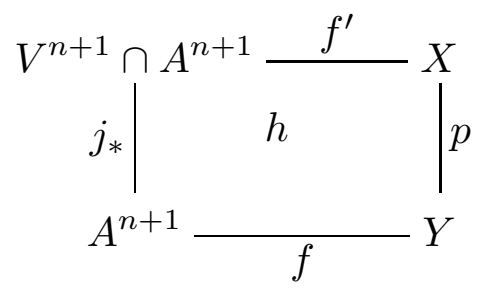

commute, since $j_{*}$ is a cofibration of simplicial sets and $p$ is a trivial fibration. The maps $\Omega h \circ \sigma_{*}: A^{n} \rightarrow \Omega X$ and $g^{\prime}: V^{n} \rightarrow \Omega X$ together induce a morphism $H: V^{n} \cup A^{n} \rightarrow \Omega X$, and there is a commutative diagram

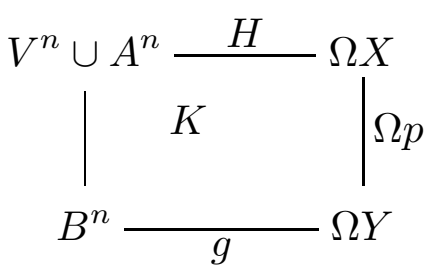

since $\Omega p$ is a trivial fibration. The map

$$
\left(K_{*}, h\right): S^{1} \wedge B^{n} \cup_{S^{1} \wedge A^{n}} A^{n+1} \rightarrow X
$$

solves the lifting problem posed by the diagram (3.4), so that $j_{*}$ has the left lifting property with respect to all trivial fibrations, and is therefore a cofibration.

Observe that the class of cofibrations satisfies analogues of the axioms sE4 sE6 (ie. without the "sE-trivial" condition), because they are defined pointwise, and cofibrations of ordinary spectra fit into a (strict) closed model structure - one could also argue directly. 
Say that a morphism $p: X \rightarrow Y$ is an sE-fibration if it has the right lifting property with respect to all $\mathbf{s E}$-trivial cofibrations. An $\mathbf{s E}$-weak equivalence is a member of the class $\mathbf{s E .}$

If $K$ is a simplicial set and $X$ is a presheaf of spectra, then the tensor object $X \ltimes K$ is defined in sections for $U \in \mathcal{C}$ by

$$
(X \ltimes K)(U)=X(U) \ltimes K=(X(U) \times K) /(* \times K)=X(U) \wedge K_{+},
$$

where $K_{+}=K \sqcup *$ is a copy of $K$ with a disjoint base point attached. For presheaves of spectra $X$ and $Y$, the function complex $\operatorname{hom}(X, Y)$ is the simplicial set whose set of $n$-simplices is defined by

$$
\operatorname{hom}(X, Y)_{n}=\operatorname{hom}\left(X \ltimes \Delta^{n}, Y\right)
$$

where the morphism set on the right is in the category of presheaves of spectra on $\mathcal{C}$. The ordinary exponential law for spectra induces a simplicial category structure on $\operatorname{Spt} \operatorname{Pre}(\mathcal{C})$.

\section{THEOREM 3.5.}

(1) Under the conditions $\mathbf{s E 1 ~ - ~ s E 7 ~ l i s t e d ~ a b o v e , ~ t h e r e ~ i s ~ a ~ c l o s e d ~ m o d e l ~ s t r u c - ~}$ ture on $\mathbf{S p t} \operatorname{Pre}(\mathcal{C})$ such that the cofibrations are the (pointwise) cofibrations as described above, the weak equivalences are the $\mathbf{s E - w e a k ~ e q u i v a l e n c e s , ~ a n d ~}$ fibrations (ie. sE-fibrations) are defined by a right lifting property.

(2) Suppose further that, given an inclusion $i: K \rightarrow L$ of finite simplicial sets and a cofibration $j: X \hookrightarrow Y$, then the induced map

$$
X \ltimes L \cup_{X \ltimes K} Y \ltimes K \hookrightarrow Y \ltimes L
$$

is an $\mathbf{s E}$-trivial cofibration if either $i$ is a weak equivalence of simplicial sets or $j$ is an $\mathbf{s E}$-weak equivalence of presheaves of spectra. Then $\mathbf{S p t} \operatorname{Pre}(\mathcal{C})$ has the structure of a closed simplicial model category.

ProOF: Suppose that $\alpha$ is an infinite cardinal which is an upper bound for the cardinality of the set of morphisms of the site $\mathcal{C}$.

The proof is analogous to the argument for Theorem 1.1, except that we need Lemma 3.1 for the proof of the factorization axiom CM5. One further shows, via the techniques in the proof of Theorem 1.1, that a map has the right lifting property with respect to all cofibrations if and only if it has the right lifting property with respect to all $\alpha$-bounded cofibrations. Then a transfinite small object argument is used to show that every map $f: X \rightarrow Y$ of presheaves of spectra has a factorization

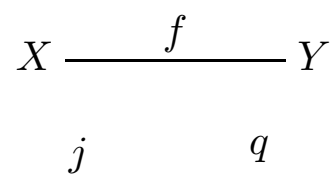


where $j$ is a cofibration, and $q$ has the right lifting property with respect to all cofibrations. In particular, the maps $q: W^{m} \rightarrow Y^{m}$ have the right lifting property with respect to all cofibrations $\partial \Delta^{n} \subset \Delta^{n}$, since the induced maps of presheaves of spectra

$$
\Sigma^{\infty}\left(L_{U} \partial \Delta^{n}\right)_{+} \rightarrow \Sigma^{\infty}\left(L_{U} \Delta^{n}\right)_{+}
$$

are cofibrations. It follows that the maps $q: W^{m} \rightarrow Y^{m}$ are pointwise weak equivalences, so that $q: W \rightarrow Y$ is a pointwise stable equivalence and therefore an $\mathbf{s E}$-weak equivalence as well as an $\mathbf{s E - f i b r a t i o n , ~ b y ~} \mathbf{s E 3}$.

REMARK 3.6. The proof of Theorem 3.5 does not assume the standard closed model structure for presheaves of spectra. This structure appears as a consequence of Theorem 3.5 in Theorem 3.7 below.

Suppose that $X$ and $Y$ are pointed simplicial sets, and that $K$ is an arbitrary simplicial set. The canonical isomorphism

$$
\operatorname{hom}(K, X) \times \operatorname{hom}(K, Y) \stackrel{\cong}{\longrightarrow} \operatorname{hom}(K, X \times Y)
$$

induces a map

$$
\operatorname{hom}(K, X) \wedge \operatorname{hom}(K, Y) \stackrel{\phi}{\rightarrow} \operatorname{hom}(K, X \wedge Y)
$$

which is natural in $K, X$ and $Y$. It follows that the maps

$$
\operatorname{hom}\left(\operatorname{sd}^{n} \Delta^{m}, X\right) \wedge \operatorname{hom}\left(\operatorname{sd}^{n} \Delta^{m}, Y\right) \stackrel{\phi}{\rightarrow} \operatorname{hom}\left(\operatorname{sd}^{n} \Delta^{m}, X \wedge Y\right)
$$

induce a pointed simplicial set map

$$
\operatorname{Ex}^{\infty} X \wedge \operatorname{Ex}^{\infty} Y \stackrel{\phi}{\rightarrow} \operatorname{Ex}^{\infty}(X \wedge Y)
$$

which is natural in $X$ and $Y$. In particular, any pointed simplicial set map

$$
S^{1} \wedge X \stackrel{\sigma}{\rightarrow} Y
$$

induces a composite pointed map

$$
S^{1} \wedge \mathrm{Ex}^{\infty} X \stackrel{\nu \wedge 1}{\longrightarrow} \operatorname{Ex}^{\infty} S^{1} \wedge \mathrm{Ex}^{\infty} X \stackrel{\phi}{\longrightarrow} \operatorname{Ex}^{\infty}\left(S^{1} \wedge X\right) \stackrel{\mathrm{Ex}^{\infty} \sigma}{\longrightarrow} \mathrm{Ex}^{\infty} Y,
$$

which will be denoted by $\tilde{\sigma}$. Here, $\nu$ is a special case of Kan's natural weak equivalence $\nu: Z \rightarrow \operatorname{Ex}^{\infty} Z$

It follows that, if $X$ is a spectrum with bonding maps $\sigma: S^{1} \wedge X^{n} \rightarrow X^{n+1}$, then there is a spectrum $\operatorname{Ex}^{\infty} X$ consisting of the pointed spaces $\operatorname{Ex}^{\infty} X^{n}$, and with bonding maps given by the induced maps $\tilde{\sigma}: S^{1} \wedge \mathrm{Ex}^{\infty} X^{n} \rightarrow \operatorname{Ex}^{\infty} X^{n+1}$. 
The spectrum $\mathrm{Ex}^{\infty} X$ is strictly fibrant (ie. consists of Kan complexes) and that the maps $\nu: X^{n} \rightarrow \operatorname{Ex}^{\infty} X^{n}$ define a strict weak equivalence of spectra $\nu: X \rightarrow$ $\operatorname{Ex}^{\infty} X$.

Suppose, generally, that $Z$ is a presheaf of spectra such that each of the objects $Z^{n}$ is a presheaf of Kan complexes. Then $Z$ has sheaves of stable homotopy groups $\pi_{i} Z$, defined for $i \in \mathbb{Z}$, where $\pi_{i} Z$ is the colimit in the sheaf category of the system of maps

$$
\pi_{i} Z^{n+i} \rightarrow \pi_{i+1} Z^{n+i+1} \rightarrow \ldots
$$

of sheaves of ordinary homotopy groups, which is, in turn, induced by the diagram of simplicial presheaf maps

$$
Z^{0} \stackrel{\sigma}{\longrightarrow} \Omega Z^{1} \stackrel{\Omega \sigma}{\longrightarrow} \Omega^{2} Z^{2} \stackrel{\Omega^{2} \sigma}{\longrightarrow} \ldots
$$

The homotopy group sheaf $\pi_{i} Z^{n+i}$ is, by definition, the sheaf associated to the presheaf $U \mapsto \pi_{i}\left(Z^{n+i}(U), *\right)$, where $*$ denotes the implicit global choice of base point. We say that a map $f: X \rightarrow Y$ is a local stable equivalence if $f$ induces isomorphisms $\pi_{i} \operatorname{Ex}^{\infty} X \cong \pi_{i} \operatorname{Ex}^{\infty} Y$ for all $i \in \mathbb{Z}$.

Suppose again that $Z$ is a presheaf of spectra which consists of presheaves of Kan complexes. The presheaf of spectra $Q Z$ has space at level $n$ defined by the filtered colimit

$$
Z^{n} \stackrel{\sigma_{*}}{\longrightarrow} \Omega Z^{n+1} \stackrel{\Omega \sigma_{*}}{\longrightarrow} \Omega^{2} Z^{n+2} \stackrel{\Omega^{2} \sigma_{*}}{\longrightarrow} \ldots,
$$

with bonding map $\sigma: Q Z^{n} \rightarrow \Omega Q Z^{n+1}$ induced by the maps $\Omega^{k} \sigma: \Omega^{k} Z^{n+k} \rightarrow$ $\Omega^{k+1} Z^{n+k+1}$. A cofinality argument implies that all bonding maps $\sigma: Q Z^{n} \rightarrow$ $\Omega Q Z^{n+1}$ are isomorphisms of simplicial presheaves, and that the canonical map $Z \rightarrow Q Z$ induces an isomorphism of presheaves of stable homotopy groups, and hence is a local stable equivalence. Finally, the canonical map

$$
\pi_{i} Q Z^{n} \rightarrow \pi_{n-i} Q Z
$$

is an isomorphism for all $n \geq 0$ and $i \geq 0$, so that a map $f: X \rightarrow Y$ of presheaves of spectra is a local stable equivalence if and only if all induced maps $f_{*}: \pi_{i} Q \mathrm{Ex}^{\infty} X^{n} \rightarrow \pi_{i} Q \mathrm{Ex}^{\infty} Y^{n}$ of sheaves of (ordinary) homotopy groups are isomorphisms.

In particular, all the morphisms $f_{*}: Q \mathrm{Ex}^{\infty} X^{n} \rightarrow Q \operatorname{Ex}^{\infty} Y^{n}$ are local weak equivalences. This is proved with a Boolean localization argument: if $p: \operatorname{Shv}(\mathcal{B}) \rightarrow$ $S h v(\mathcal{C})$ is a Boolean localization, then each of induced map

$$
p^{*} f_{*}: p^{*} L^{2} Q \operatorname{Ex}^{\infty} X^{n}(b) \rightarrow p^{*} L^{2} Q \operatorname{Ex}^{\infty} Y^{n}(b)
$$

in sections is a map of Kan complexes which are also loop spaces, and this map preserves the loop space structure. Furthermore, this map induces an isomorphism

$$
\pi_{i} p^{*} L^{2} Q \operatorname{Ex}^{\infty} X^{n}(b) \cong \pi_{i} p^{*} L^{2} Q \operatorname{Ex}^{\infty} X^{n}(b),
$$


for $i \geq 0$, since Boolean localization commutes with the formation of all sheaves of homotopy groups (see [15]), and so the ambient $\mathrm{H}$-space structures can be used to show that it is a weak equivalence, in all sections.

Conversely, or at least partially so, if $f: X \rightarrow Y$ is a map of presheaves of spectra such that each of the maps $f: X^{n} \rightarrow Y^{n}$ is a local weak equivalence, then $f$ induces an isomorphism in sheaves of stable homotopy groups.

The following result was first proved in [13]. We give here a new "one step" proof which is based on Theorem 3.5. The proof of this result specializes to an alternative demonstration of the existence of the Bousfield-Friedlander stable closed model structure for ordinary spectra [5]. Note, however, that we still require the strict closed model structure for ordinary spectra to take care of some of the standard assertions about cofibrations. The existence of the strict structure is just an exercise.

Theorem 3.7. Say that a map $f: X \rightarrow Y$ of presheaves of spectra on a small site $\mathcal{C}$ is a local stable equivalence if it induces an isomorphism in all sheaves of stable homotopy groups, and that a map is a global fibration if it has the right lifting property with respect to all maps which are cofibrations and local stable equivalences. Then with these definitions, the category $\operatorname{Spt} \operatorname{Pre}(\mathcal{C})$ of presheaves of spectra on a Grothendieck site $\mathcal{C}$ satisfies the axioms for a proper closed simplicial model category.

Proof: We verify the conditions of Theorem 3.5 to show that the category of presheaves of spectra on $\mathcal{C}$ is a closed simplicial model category where, in the notation of that result, $\mathbf{s E}$ stands for the class of local stable equivalences.

The stable homotopy group functor preserves filtered colimits and takes wedges to direct sums, so the only axiom having any content is $\mathbf{s E 7}$. Suppose that $\alpha$ is an infinite cardinal which is a strict upper bound for the cardinality of the set of morphisms of the underlying site, and suppose given a diagram

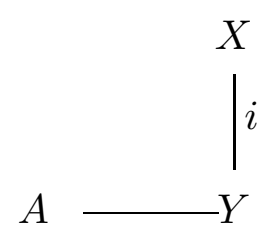

where $A$ is an $\alpha$-bounded subobject of $Y$ and $i$ is a cofibration which is a local stable equivalence. This means in particular that the presheaves of stable homotopy groups $\pi_{i}^{p} Y / X$ are locally trivial in the sense that any section $x \in \pi_{i}^{p} Y / X(U)$ maps to 0 along some covering sieve $R \subset \operatorname{hom}(, U)$ in the sense that $\phi^{*}(x)=0$ for all $\phi: V \rightarrow U$ in $R$. The presheaf of spectra $Y$ is a filtered colimit of its $\alpha$-bounded subobjects, so it follows that any $x \in \pi_{*}^{p}(A / A \cap X)(U)$ maps to 0 in $\pi_{*}^{p}\left(A_{1} / A_{1} \cap X\right)$ 
for some $\alpha$-bounded $A_{1}$ with $A \subset A_{1} \subset Y$. Continue inductively, to produce a sequence of $\alpha$-bounded subobjects

$$
A=A_{0} \subset A_{1} \subset A_{2} \subset \ldots
$$

of $Y$ such that the induced maps

$$
\pi_{*}^{p}\left(A_{i} / A_{i} \cap X\right) \rightarrow \pi_{*}^{p}\left(A_{i+1} / A_{i+1} \cap X\right)
$$

of presheaves of stable homotopy groups are all 0-maps. Let $B=\cup_{i \geq 0} A_{i}$. Then $B$ is an $\alpha$-bounded subobject of $Y$ containing $A$ such that $\pi_{*}^{p}(B / B \cap X)=0$, so that the cofibration $B \cap X \hookrightarrow B$ is a local stable equivalence.

The class of maps which are both cofibrations and local stable equivalences is closed under pushout, by a standard long exact sequence argument. Suppose that $i: K \hookrightarrow L$ is an inclusion of finite simplicial sets and that $j: X \rightarrow Y$ is a cofibration of presheaves of spectra. Then the induced cofibration

$$
X \ltimes L \cup_{X \ltimes K} Y \ltimes K \hookrightarrow Y \ltimes L
$$

is a local stable equivalence if either $i$ is a weak equivalence or $j$ is a local stable equivalence. In effect, if $i$ is a weak equivalence, then the maps $X \ltimes i$ and $Y \ltimes i$ are strict and hence local stable equivalences, as is the pushout map

$$
Y \ltimes K \stackrel{i_{*}}{\longrightarrow} X \ltimes L \cup_{X \ltimes K} Y \ltimes K .
$$

For the other case, if $i: X \rightarrow Y$ is a local stable equivalence, then the maps

$$
i_{*}: \bigvee_{\sigma \in K_{n}} X \rightarrow \bigvee_{\sigma \in K_{n}} Y
$$

are local stable equivalences, so that the map $i: X \ltimes K \rightarrow Y \ltimes K$ is a local stable equivalence, by a spectral sequence argument (see [14]). This holds for every simplicial set $K$, and in particular for $L$.

Every global fibration $p: X \rightarrow Y$ is a strict fibration, in the sense that all maps $p: X^{n} \rightarrow Y^{n}$ are global fibrations of simplicial presheaves, since the functors $U \mapsto \Sigma^{\infty} U_{+}[-n]$ take trivial cofibrations of simplicial presheaves to cofibrations of presheaves of spectra which are local stable equivalences. Suppose that the diagram

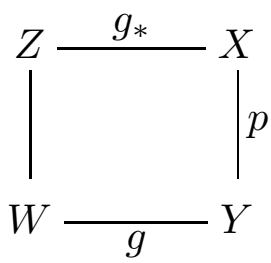


is a pullback in the category of presheaves of spectra, where $p$ is a global fibration and $g$ is a local stable equivalence. We want to show that the map $g_{*}$ is a local stable equivalence. Each induced diagram

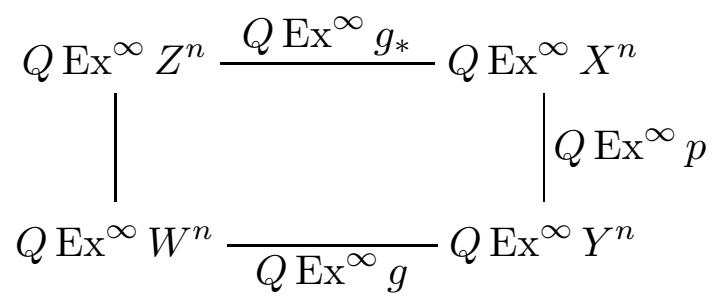

is a pullback diagram of simplicial presheaves in which all the objects are presheaves of Kan complexes, the map $Q \mathrm{Ex}^{\infty} g$ is a local weak equivalence, and the map $Q \mathrm{Ex}^{\infty} p$ is a local fibration. We saw in the proof of Theorem 2.7 that local weak equivalences are stable under pullback along local fibrations, so that $Q \mathrm{Ex}^{\infty} g_{*}$ is a local weak equivalence, and so the map $g_{*}$ is a local stable equivalence.

One shows that local stable equivalences are preserved by pushing out along cofibrations by comparing long exact sequences for cofibrations.

Theorem 3.9. Suppose that $F: \mathcal{C} \rightarrow \mathcal{D}$ is a continuous functor between small Grothendieck sites which induces a geometric morphism $\operatorname{Shv}(\mathcal{D}) \rightarrow \operatorname{Shv}(\mathcal{C})$, and that $E$ is a presheaf of spectra on $\mathcal{D}$. Say that a map $g: X \rightarrow Y$ of simplicial presheaves on $\mathcal{C}$ is an $E_{*}$-weak equivalence if the induced map $g_{*}: E \wedge F^{p} X_{+} \rightarrow$ $E \wedge F^{p} Y_{+}$is a local stable equivalence of presheaves of spectra on $\mathcal{D}$. Say that a map $p: Z \rightarrow W$ is an $E_{*}$-global fibration if $p$ has the right lifting property with respect to all cofibrations which are $E_{*}$-weak equivalences. Then the classes of cofibrations, $E_{*}$-weak equivalences and $E_{*}$-global fibrations give the category $\mathbf{S} \operatorname{Pre}(\mathcal{C})$ the structure of a closed simplicial model category.

Proof: The functor $X \mapsto E \wedge F^{p} X_{+}$takes values in the category of presheaves of spectra on the site $\mathcal{D}$, which is a closed simplicial model category by the main result of [13], or Theorem 3.7. The notation $F^{p} X_{+}$, as usual, denotes $F^{p} X$ with a disjoint base point attached. We shall prove this result by invoking Theorem 1.1, where the class $\mathbf{E}$ is the collection of $E_{*}$-weak equivalences.

We can assume that the presheaf of spectra $E$ is cofibrant, because otherwise there is a trivial strict fibration $\pi: E^{\prime} \rightarrow E$, where $E^{\prime}$ is cofibrant, and the induced map $\pi \wedge 1: E^{\prime} \wedge F^{p} X_{+} \rightarrow E \wedge F^{p} X_{+}$is a strict and hence local stable weak equivalence for all $X$, so that a map $g: X \rightarrow Y$ of simplicial presheaves on $\mathcal{C}$ is an $E_{*}$-weak equivalence if and only if it is an $E_{*}^{\prime}$-weak equivalence.

If $i: A \rightarrow B$ is a cofibration of $\mathbf{S} \operatorname{Pre}(\mathcal{C})$, then the induced map $F^{p}(i): F^{p} A_{+} \rightarrow$ $F^{p} B_{+}$is canonically locally weakly equivalent to a natural choice of cofibration, 
namely the $L^{2} F^{p}(i): L^{2} F^{p} A_{+} \rightarrow L^{2} F^{p} B_{+}$. In particular, if the diagram

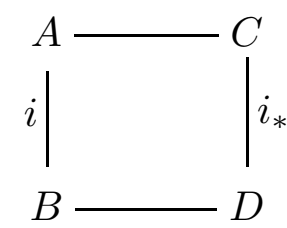

is a pushout diagram of simplicial presheaves on the site $\mathcal{C}$, where $i$ is an $E_{*}$-trivial cofibration, then the pushout diagram

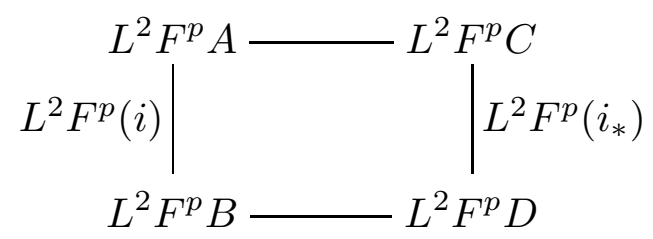

of simplicial sheaves on $\mathcal{D}$ contains a cofibration $L^{2} F^{p}(i)$ which induces a local stable equivalence

$$
E \wedge L^{2} F^{p}(i)_{+}: E \wedge L^{2} F^{p} A_{+} \rightarrow E \wedge L^{2} F^{p} B_{+} .
$$

Cofibrations of $\mathbf{S} \operatorname{Pre}(\mathcal{D})$ which induce a local stable equivalence after smashing with $E$ are stable under pushout, and so the same is true for cofibrations of simplicial sheaves on $\mathcal{D}$, since the associated sheaf functor preserves pushouts. It follows that the class of $E_{*}$-trivial cofibrations of $\mathbf{S} \operatorname{Pre}(\mathcal{C})$ is closed under pushout.

We used a piece of Lemma 2.6 implicitly in this last argument: if $f: X \rightarrow Y$ is a pointwise weak equivalence, then the simplicial presheaf map $f_{*}: F^{p} X_{+} \rightarrow F^{p} Y_{+}$ on $\mathcal{D}$ is a local weak equivalence. It follows that the induced map $f_{*}: E \wedge F^{p} X_{+} \rightarrow$ $E \wedge F^{p} Y_{+}$is a strict and hence local stable equivalence, so that every pointwise equivalence is an $E_{*}$-weak equivalence, giving E3.

With the exception of E7, the remaining axioms have trivial proofs. It remains to verify the bounded cofibration condition.

Let $\alpha$ be an infinite cardinal which is an upper bound on the cardinalities of the morphisms set of $\mathcal{C}$, the morphism set of $\mathcal{D}$, and all sets of sections of all simplicial presheaves $E^{n}, n \geq 0$ making up the presheaf of spectra $E$. Suppose that we are given a diagram of simplicial presheaf maps

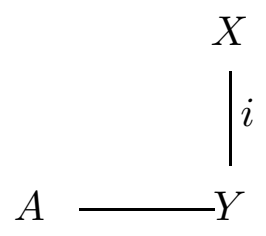


on $\mathcal{C}$, where $i$ is a cofibration which is an $E_{*}$-equivalence, and $A$ is an $\alpha$-bounded subobject of $Y$. The assertion that $i$ is an $E_{*}$-equivalence means exactly that all stable homotopy group presheaves $\pi_{i}^{p}\left(E \wedge F^{p}(Y / X)\right)$ are locally trivial in the sense that every section $x \in \pi_{i}^{p}\left(E \wedge F^{p}(Y / X)\right)(U)$ maps to 0 along all maps in some covering sieve $R \subset \operatorname{hom}(, U)$, and for all objects $U$ of the site $\mathcal{D}$. The presheaves of stable homotopy groups $\pi_{i}^{p}\left(E \wedge F^{p}(A /(A \cap X))\right.$ are $\alpha$-bounded, by the choice of the size of the cardinal $\alpha$. The functor $B \mapsto \pi_{i}^{p}\left(E \wedge F^{p} B\right)$ preserves filtered colimits in pointed simplicial presheaves $B$ on $\mathcal{C}$, and $Y$ is a filtered colimit of its $\alpha$-bounded subobjects. It follows that there is an $\alpha$-bounded subobject $A_{1}$ of $Y$ such that $A \subset A_{1}$, and such that the induced map of presheaves of stable homotopy groups

$$
\pi_{i}^{p}\left(E \wedge F^{p}(A /(A \cap X))\right) \rightarrow \pi_{i}^{p}\left(E \wedge F^{p}\left(A_{1} /\left(A_{1} \cap X\right)\right)\right)
$$

is the zero map in all sections, and for all $i$. Repeating this construction inductively gives a sequence of inclusions

$$
A=A_{0} \subset A_{1} \subset A_{1} \subset \ldots
$$

of $\alpha$-bounded subobjects of $Y$ such that all induced maps

$$
\pi_{i}^{p}\left(E \wedge F^{p}\left(A_{j} /\left(A_{j} \cap X\right)\right)\right) \rightarrow \pi_{i}^{p}\left(E \wedge F^{p}\left(A_{j+1} /\left(A_{j+1} \cap X\right)\right)\right)
$$

of presheaves of stable homotopy groups are 0 . Let $B=\cup_{i} A_{i}$ : then $B$ is an $\alpha$ bounded subobject of $Y$ containing $A$ such that $\pi_{i}\left(E \wedge F^{p}(B /(B \cap X))\right)=0$ for all $i$, so that the inclusion $B \cap X \hookrightarrow B$ is an $E_{*}$-equivalence.

The natural simplicial set isomorphism

$$
K_{+} \ltimes L \cong(K \times L)_{+}
$$

implies that the functor $X \mapsto E \wedge F^{p} X_{+}$is a simplicial functor which preserves tensors, along with pushouts and cofibrations. It follows that if $i: X \hookrightarrow Y$ is a cofibration of simplicial presheaves on $\mathcal{C}$ and $j: K \hookrightarrow L$ is an inclusion of finite simplicial sets, then the induced cofibration

$$
(X \times L) \cup_{(X \times K)}(Y \times K) \hookrightarrow Y \times L
$$

is an $E_{*}$-weak equivalence if either $i$ is an $E_{*}$-weak equivalence or $j$ is a trivial cofibration of simplicial sets.

We say that the closed model structure arising from the presheaf of spectra $E$ and the functor $F: \mathcal{C} \rightarrow \mathcal{D}$ is the $E_{*}$-theory. An $E_{*}$-fibrant model $X \rightarrow L_{E} X$ is an $E_{*}$-weak equivalence such that $L_{E} X$ is $E_{*}$-fibrant. Such things always exist, and any two $E_{*}$-fibrant models for $X$ are non-canonically homotopy equivalent, 
by the Whitehead Theorem. An $E_{*}$-fibrant model for $X$ is also said to be an $E_{*}$-localization of $X$.

Recall from [14] that a bispectrum $X$ can be defined to be a spectrum object in the category of spectra, in the sense that $X$ consists of spectra $X^{n}, n \geq 0$, and maps of spectra $\sigma: X^{n} \wedge S^{1} \rightarrow X^{n+1}$. The maps $\sigma$ are usually called bonding maps. A morphism $i: X \rightarrow Y$ of bispectra is a cofibration if the map $i^{0}: X^{0} \rightarrow Y^{0}$ at level 0 is a cofibration of ordinary spectra, along with all induced maps

$$
\left(Y^{n} \wedge S^{1}\right) \cup_{\left(X^{n} \wedge S^{1}\right)} X^{n+1} \rightarrow Y^{n+1}
$$

The spectra $Y^{n}$ themselves consist of spaces $Y^{n, k}, k \geq 0$, and there is a diagonal spectrum $d(Y)$ associated to the bispectrum $Y$, such that $d(Y)^{2 r}=Y^{r, r}$, $d(Y)^{2 r+1}=Y^{r, r+1}$, and such that the bonding maps are given by the pointed simplicial set maps

$$
S^{1} \wedge Y^{r, r} \rightarrow Y^{r, r+1}
$$

and the composites

$$
S^{1} \wedge Y^{r, r+1} \underset{\stackrel{\tau}{\cong}}{\cong} Y^{r, r+1} \wedge S^{1} \rightarrow Y^{r+1, r+1}
$$

arising from the spectrum structure for $Y^{r}$ and the bispectrum structure for $Y$, respectively. A map $f: X \rightarrow Y$ of bispectra is said to be a stable equivalence if the induced map $f_{*}: d(X) \rightarrow d(Y)$ is a stable equivalence of ordinary spectra.

These definitions generalize quite naturally to presheaves of bispectra. A map $f: X \rightarrow Y$ of presheaves of bispectra is said to be a cofibration if each of the maps $f: X(U) \rightarrow Y(U), U \in \mathcal{C}$, in sections of ordinary bispectra is a cofibration in the sense described above. The map $f: X \rightarrow Y$ is said to be a local stable equivalence if the induced map $f_{*}: d(X) \rightarrow d(Y)$ of diagonal presheaves of spectra is a local stable equivalence. Write $\mathbf{S p t}^{2} \operatorname{Pre}(\mathcal{C})$ for the category of presheaves of bispectra on the site $\mathcal{C}$. We shall use the result, proved in [14], that the category $\mathbf{S p t}^{2} \operatorname{Pre}(\mathcal{C})$ has a proper closed simplicial model structure for which the weak equivalences are the local stable equivalences and the cofibrations are as above.

TheOrem 3.10. Suppose that $F: \mathcal{C} \rightarrow \mathcal{D}$ is a continuous functor between small Grothendieck sites which induces a geometric morphism $S h v(\mathcal{D}) \rightarrow \operatorname{Shv}(\mathcal{C})$, and that $E$ is a presheaf of spectra on $\mathcal{D}$. Say that a map $f: X \rightarrow Y$ of presheaves of spectra on $\mathcal{C}$ is an $E_{*}$-weak equivalence if the induced map $f_{*}: F^{p} X \wedge E \rightarrow F^{p} Y \wedge E$ is a local stable equivalence of presheaves of bispectra on $\mathcal{D}$. Say that a map $p: Z \rightarrow W$ is an $E_{*}$-global fibration if $p$ has the right lifting property with respect to all cofibrations which are $E_{*}$-weak equivalences. Then the classes of cofibrations, $E_{*}$-weak equivalences and $E_{*}$-global fibrations give the category $\operatorname{Spt} \operatorname{Pre}(\mathcal{C})$ the structure of a closed simplicial model category. 
Proof: We can assume that the spectrum $E$ is cofibrant, by replacing it up to strict weak equivalence by a cofibrant object. Axioms sE1 and sE2 are trivial.

If the map $g: Z \rightarrow W$ of presheaves of spectra on $\mathcal{C}$ is a pointwise strict weak equivalence, then each level map $g: Z^{n} \rightarrow W^{n}$ is a local weak equivalence of simplicial presheaves, so that each map $F^{p}(g): F^{p} Z^{n} \rightarrow F^{p} W^{n}$ is a local weak equivalence of simplicial presheaves on $\mathcal{D}$. But then each of the induced maps $F^{p} Z^{n} \wedge E^{m} \rightarrow F^{p} W^{n} \wedge E^{m}$ is a local weak equivalence of simplicial presheaves on $\mathcal{D}$, so that $g_{*}: F^{p} Z \wedge E \rightarrow F^{p} W \wedge E$ is a local stable equivalence of presheaves of bispectra on $\mathcal{D}$, giving sE3.

Suppose given a pushout diagram

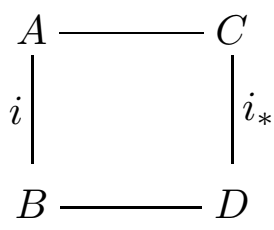

of presheaves of spectra on the site $\mathcal{C}$ for which the map $i$ is a cofibration and an $E_{*}$-weak equivalence. In order to prove $\mathbf{s E 4}$, it suffices to show that the map $i_{*}$ is an $E_{*}$-weak equivalence. There is an induced pushout diagram

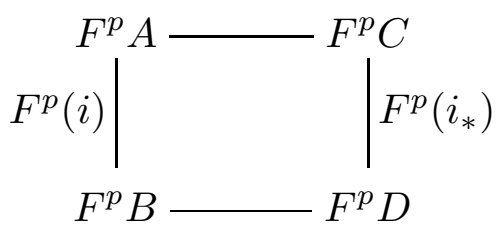

of presheaves of spectra on $\mathcal{D}$. The map $F^{p}(i)$ may not be a cofibration, but it induces a map $L^{2} F^{p}(i): L^{2} F^{p} A \rightarrow L^{2} F^{p} B$ which is a pointwise cofibration [14, Ch.2] in the sense that all level maps $L^{2} F^{p}(i): L^{2} F^{p} A^{n} \rightarrow L^{2} F^{p} B^{n}$ are cofibrations of simplicial (pre-) sheaves, after applying the associated sheaf functor, by exactness of the inverse image functor $F^{*}: S h v(\mathcal{C}) \rightarrow S h v(\mathcal{D})$. Furthermore, the induced map $E \wedge L^{2} F^{p} A \rightarrow E \wedge L^{2} F^{p} B$ is a local stable equivalence of presheaves of bispectra on $\mathcal{D}$, since it is strictly locally weakly equivalent to the map $E \wedge F^{p} A \rightarrow E \wedge F^{p} B$. The collection of pointwise cofibrations of presheaves of spectra on $\mathcal{D}$ which induce a local stable equivalence of presheaves of bispectra after smashing with $E$ is closed under pushout, by a standard long exact sequence argument. It follows that the map $i_{*}$ induces local stable equivalences $E \wedge L^{2} F^{p} C \rightarrow E \wedge L^{2} F^{p} D$ and $E \wedge F^{p} C \rightarrow$ $E \wedge F^{p} D$. 
Axioms sE5 and sE6 are easily verified. The proof of the bounded cofibration axiom sE7 proceeds just as in the proof of Theorem 3.9.

Note the use of sheaves of spectra in the proof of Theorem 3.10. In general, a sheaf of spectra $Y$ is a presheaf of spectra such that all of the objects $Y^{n}, n \geq 0$, are simplicial sheaves.

If $X$ is a pointed simplicial presheaf on a site $\mathcal{C}$ and $K$ is a pointed simplicial set, there is a natural canonical map

$$
\eta_{*}: K \wedge L^{2} X \rightarrow L^{2}(K \wedge X)
$$

which respects the associated sheaf maps $\eta: X \rightarrow L^{2} X$ and $\eta: K \wedge X \rightarrow L^{2}(K \wedge X)$ in the obvious way. It follows that, given a presheaf of spectra $Z$, there is an associated sheaf of spectra $L^{2} Z$ having bonding maps given by the composites

$$
S^{1} \wedge L^{2} Z^{n} \stackrel{\eta_{*}}{\longrightarrow} L^{2}\left(S^{1} \wedge Z^{n}\right) \stackrel{L^{2}(\sigma)}{\longrightarrow} L^{2} Z^{n+1} .
$$

This construction is functorial in $Z$, and there is a natural map of presheaves of spectra $\eta: Z \rightarrow L^{2} Z$ which consists of the associated sheaf map $\eta: Z^{n} \rightarrow L^{2} Z^{n}$ in all levels. This map $\eta$ is a strict local weak equivalence.

REMARK 3.11. There is a stable proper closed simplicial model structure for the category $\operatorname{Spt} S h v(\mathcal{C})$, for which the weak equivalences are those maps which are local stable equivalences in the simplicial presheaf category. The cofibrations, however, are different in that they need to be defined within the category of simplicial sheaves. Explicitly, a map $i: X \rightarrow Y$ is a cofibration of sheaves of spectra if the following two conditions hold:

(1) the map $i: X^{0} \rightarrow Y^{0}$ is a cofibration of simplicial sheaves, and

(2) for each $n \geq 0$ the map

$$
L^{2}\left(\left(S^{1} \wedge Y^{n}\right) \cup_{\left(S^{1} \wedge X^{n}\right)} X^{n+1}\right) \hookrightarrow Y^{n+1}
$$

is a cofibration of simplicial sheaves.

Observe that every map of sheaves of spectra which is a cofibration of presheaves of spectra is also a cofibration of sheaves of spectra. The stable proper closed simplicial model structure for $\operatorname{Spt} S h v(\mathcal{C})$ follows from the existence of the corresponding structure for the category $\operatorname{Spt} \operatorname{Pre}(\mathcal{C})$ of presheaves of spectra on $\mathcal{C}$ (Theorem 3.7), in the same way that Theorem 1.2 follows from Theorem 1.1. Furthermore, the natural map $\eta: Z \rightarrow L^{2} Z$ is a strict local equivalence, so the stable closed model structures for presheaves of spectra and sheaves of spectra have equivalent associated homotopy categories.

The pointwise cofibration $L^{2} F^{p}(i): L^{2} F^{p} A \rightarrow L^{2} F^{p} B$ of presheaves of spectra which appears in the proof of Theorem 3.10 is actually a cofibration of sheaves of spectra, and the proof of that result can be rewritten on this basis. 
REMARK 3.12. Theorem 3.10 specializes to a closed model structure for ordinary spectra, where $E$ is any spectrum, and the weak equivalences are those maps which induce stable equivalences after smashing with $E$.

\section{Localization with respect to a map.}

Let $\mathbf{S}$ be the category of simplicial sets and $f: A \rightarrow B$ a cofibration. A great deal of thought has gone into the notion of an $f$-localization. We refer the reader especially to the work of Dror-Farjoun ([6], [7]), Hirschhorn [10], and Bousfield [4]. All of these contain further references and applications. We begin this section by presenting a synopsis of the theory.

A simplicial set $X$ is $f$-local if it is fibrant and if the fibration

$$
f^{*}: \operatorname{hom}(B, X) \rightarrow \operatorname{hom}(A, X)
$$

is a weak equivalence. An $f$-localization of $Y \in \mathbf{S}$ is a morphism $Y \rightarrow X$ in $\mathbf{S}$ to an $f$-local object which is initial among all such morphisms; that is, given a map $Y \rightarrow Z$ to a $f$-local object there is a unique homotopy class of maps $X \rightarrow Z$ making the following triangle commute in the homotopy category:

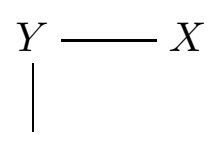

$Z$.

One of the main results of the work cited above is that localizations exist and induce a functor $L_{f}: \operatorname{Ho}(\mathbf{S}) \rightarrow \mathrm{Ho}(\mathbf{S})$ which is augmented in the sense that there is a natural map $\eta_{X}: X \rightarrow L_{f} X$ and idempotent in the sense that the two natural maps

$$
L_{f} \eta_{X}, \eta_{L_{f} X}: L_{f} X \rightarrow\left(L_{f}\right)^{2} X
$$

are equal and isomorphisms in $\operatorname{Ho}(\mathbf{S})$.

The functor $L_{f}: \operatorname{Ho}(\mathbf{S}) \rightarrow \operatorname{Ho}(\mathbf{S})$ is induced by a functor $\mathcal{L}=\mathcal{L}_{f}: \mathbf{S} \rightarrow \mathbf{S}$. To describe $\mathcal{L}$, consider the set $\mathbf{C}$ of cofibrations with elements

$$
A \times \Delta^{n} \cup_{A \times \partial \Delta^{n}} B \times \partial \Delta^{n} \rightarrow B \times \Delta^{n}, \quad n \geq 0 .
$$

Since $\mathbf{C}$ is a set, there is an infinite cardinal $\alpha$ so that the source and target of all morphisms in $\mathbf{C}$ are $\alpha$-bounded.

The space $\mathcal{L} X$ is defined by a filtered colimit

$$
\mathcal{L} X=\underset{s<\kappa}{\lim _{s<}} E_{s} X,
$$


where $\kappa$ is some fixed cardinal with $\kappa>2^{\alpha}$, and $E_{s} X$ is defined by transfinite induction. $E_{0} X$ is a functorial choice of fibrant model for $X$ - it is most convenient here to write $E_{0} X=\operatorname{Ex}^{\infty} X$. If $s$ is a limit ordinal $E_{s}=\lim _{t<s} E_{t}$, and for successor ordinals $s+1$, there is a push-out square

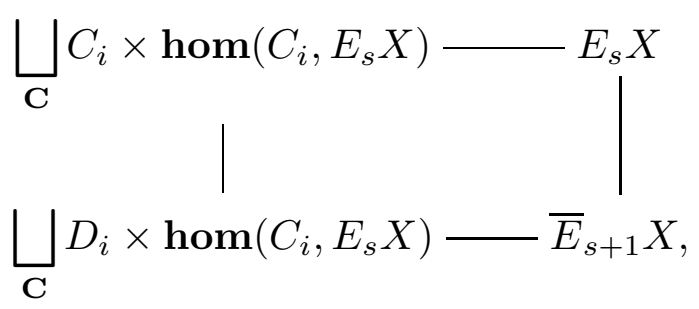

and then $E_{s+1} X=\mathrm{Ex}^{\infty} \bar{E}_{s+1} X$ is a fibrant model for $\bar{E}_{s+1} X$. We shall say that $\kappa$ is the defining cardinal for the functor $\mathcal{L}$.

The slightly expanded definition of the stages in the factorization (using function spaces rather than merely sets of maps) is required for the resulting object to be continuous in the sense of $\mathbf{L} \mathbf{7}$ below.

The canonical map $\eta_{X}: X \rightarrow \mathcal{L} X$ is a cofibration and induces a weak equivalence

$$
\eta_{X}^{*}: \operatorname{hom}(\mathcal{L} X, Z) \rightarrow \operatorname{hom}(X, Z)
$$

for all $f$-local objects $Z$, by construction. Furthermore, $\mathcal{L} X$ is $f$-local: it is clearly fibrant and any map $C_{i} \rightarrow \mathcal{L} X$ factors through some $E_{s} X$, for otherwise $C_{i}$ would have too many subobjects.

The functor $\mathcal{L}$ also satisfies the following properties.

L1: $\mathcal{L}$ preserves weak equivalences.

L2: $\mathcal{L}$ preserves cofibrations.

L3: Let $\beta$ be any cardinal with $\beta \geq \alpha$. Let $\left\{X_{j}\right\}$ be the filtered system of sub-objects of $X$ which are $\beta$-bounded. Then the map

$$
\underset{j}{\lim _{j}} \mathcal{L}\left(X_{j}\right) \rightarrow \mathcal{L}(X)
$$

is an isomorphism.

L4: Let $\gamma$ be an ordinal number of cardinality strictly greater than $2^{\alpha}$. Let $X: \gamma \rightarrow \mathbf{S}$ be a diagram of cofibrations so that for all limit ordinals $s<\gamma$ the induced map

$$
\underset{t<s}{\lim } X(t) \rightarrow X(s)
$$


is an isomorphism. Then $\lim _{t<\gamma} \mathcal{L}(X(t)) \cong \mathcal{L}\left(\underline{\lim }_{t<\gamma} X(t)\right)$.

L5: Suppose that $\lambda=2^{\kappa}$, where $\kappa$ is the defining cardinal for the functor $\mathcal{L}$. If $X$ is $\lambda$-bounded, then $\mathcal{L}(X)$ is $\lambda$-bounded.

L6: Let $Y, Z$ be two subobjects of $X$. Then

$$
\mathcal{L}(Y) \cap \mathcal{L}(Z)=\mathcal{L}(Y \cap Z)
$$

in $\mathcal{L}(X)$.

L7: The functor $\mathcal{L}$ is continuous; that is, it extends to a natural morphism of simplicial sets

$$
\mathcal{L}: \operatorname{hom}(X, Y) \rightarrow \operatorname{hom}(\mathcal{L} X, \mathcal{L} Y)
$$

compatible with composition.

The idea of proof for $\mathbf{L} \mathbf{1}$ is to show inductively that any weak equivalence $g$ : $X \rightarrow Y$ induces weak equivalences $g_{*}: E_{s} X \rightarrow E_{s} Y$. This is a patching lemma argument, given that $g$ induces a weak equivalence

$$
g_{*}: \operatorname{hom}\left(C_{i}, E_{s} X\right) \rightarrow \operatorname{hom}\left(C_{i}, E_{s} Y\right)
$$

since $E_{s} X$ and $E_{s} Y$ are fibrant.

For $\mathbf{L 2}$, one shows inductively that if $X \rightarrow Y$ is a cofibration, then $E_{s} X \rightarrow E_{s} Y$ is a cofibration and, for successor ordinals,

$$
E_{s}(Y) \cup_{E_{s}(X)} \bar{E}_{s+1}(X) \rightarrow \bar{E}_{s+1}(Y)
$$

is a cofibration. This last ultimately relies on the fact that for all $C_{i} \rightarrow D_{i}$ in $\mathbf{C}$, the map

$$
C_{i} \times \operatorname{hom}\left(C_{i}, E_{s} Y\right) \cup_{C_{i} \times \operatorname{hom}\left(C_{i}, E_{s} X\right)} D_{i} \times \operatorname{hom}\left(C_{i}, E_{s} X\right) \rightarrow D_{i} \times \operatorname{hom}\left(C_{i}, E_{s} Y\right)
$$

is an inclusion, hence a cofibration. In particular, one needs that

$$
\operatorname{hom}\left(C_{i}, E_{s} X\right) \rightarrow \operatorname{hom}\left(C_{i}, E_{s} Y\right)
$$

is an inclusion. Note further that Kan's $\mathrm{Ex}^{\infty}$ construction preserves cofibrations.

The statement $\mathbf{L} 3$ is again verified by proving the corresponding statement for each of the functors $E_{s}$. Suppose that $g: C_{i} \times \Delta^{n} \rightarrow E_{s} X$ is a map, and take a simplex $(x, \theta)$ of $C_{i} \times \Delta^{n}$. Then $g(x, \theta)$ lies in some $E_{s} Y$ such that $Y$ is a $\beta$-bounded subcomplex of $X$ by the inductive assumption. But then all images $f(x, \theta)$ lie in some $E_{s} W$ where $W$ is a $\beta$-bounded subcomplex of $X$, since $\left|C_{i} \times \Delta^{n}\right| \leq \alpha$, and 
the collection of all $\beta$-bounded subcomplexes of $X$ is closed under unions of size at most $\beta$. We have therefore seen that

$$
\underset{j}{\lim } \operatorname{hom}\left(C_{i}, E_{s} X_{j}\right) \rightarrow \operatorname{hom}\left(C_{i}, E_{s} X\right)
$$

is an isomorphism for all $C_{i} \rightarrow D_{i}$ in $\mathbf{C}$. The rest of the verification is formal, since the $\mathrm{Ex}^{\infty}$ functor preserves filtered colimits.

The statement $\mathbf{L} \mathbf{4}$ has a similar but easier argument: there is an isomorphism

$$
\underset{t<\gamma}{\lim } \operatorname{hom}\left(C_{i}, E_{s} X(t)\right) \cong \operatorname{hom}\left(C_{i}, \underset{t<\gamma}{\lim _{t<\gamma}} E_{s} X(t)\right),
$$

since otherwise $C_{i}$ would have too many subobjects.

For $\mathbf{L} \mathbf{5}$, one shows that $E_{s}(X)$ is $\lambda$-bounded, and uses the observation that

$$
\lambda=2^{\kappa}>\kappa>2^{\alpha}>\alpha
$$

The fact that $E_{s+1}(X)$ is $\lambda$-bounded ultimately relies on the fact that

$$
\left|D_{i} \times \operatorname{hom}\left(C_{i}, E_{s} X\right)\right| \leq \alpha \cdot \lambda^{\alpha}=\alpha \cdot\left(2^{\kappa}\right)^{\alpha}=\alpha \cdot 2^{\kappa \cdot \alpha}=\lambda .
$$

Note that if $Y$ is $\lambda$-bounded, then $\operatorname{Ex}^{\infty} Y$ is $\lambda$-bounded, since $\lambda$ is infinite.

Note that by $\mathbf{L} 2$ we may assume we have $\mathcal{L}(Y) \cap \mathcal{L}(Z) \subseteq \mathcal{L}(Y \cap Z) \subseteq \mathcal{L}(X)$. One shows that $E_{s}(Y) \cap E_{s}(Z)=E_{s}(Y \cap Z)$. The limit ordinal case follows from the successor ordinal case because filtered colimits commute with pullbacks. The successor ordinal case follows from fact that, degreewise, $E_{s+1} X_{n}$ has the form

$$
E_{s+1} X_{n}=\left(\bigsqcup_{C}\left(D_{i}-C_{i}\right) \times \operatorname{hom}\left(C_{i}, E_{s} X\right)_{n}\right) \sqcup E_{s} X_{n},
$$

and the image of the inclusion $E_{s} Y_{n} \hookrightarrow E_{s} X_{n}$ associated to any subcomplex $Y \subset X$ has the form

$$
\left(\bigsqcup_{C}\left(D_{i}-C_{i}\right) \times \operatorname{hom}\left(C_{i}, E_{s} Y\right)_{n}\right) \sqcup E_{s} Y_{n}
$$

The $\mathrm{Ex}^{\infty}$ functor preserves pullbacks, giving $\mathbf{L 6}$.

The statement $\mathbf{L 7}$ is equivalent to asserting that for all $X, K \in \mathbf{S}$, there is a natural map $\mathcal{L}(X) \times K \rightarrow \mathcal{L}(X \times K)$ so that the following triangle commutes

$$
\begin{array}{r}
X \times K \frac{\eta_{X} \times 1}{\eta_{X \times K}} \mathcal{L}(X) \times K \\
\mathcal{L}(X \times K)
\end{array}
$$


subject to the requirements that $\mathcal{L}(X) \times \Delta^{0} \cong \mathcal{L}\left(X \times \Delta^{0}\right)$, and the following two maps agree:

$$
\mathcal{L}(X) \times(K \times L) \rightarrow \mathcal{L}(X \times(K \times L)) \cong \mathcal{L}((X \times K) \times L))
$$

and

$$
\mathcal{L}(X) \times(K \times L) \rightarrow(\mathcal{L}(X) \times K) \times L) \rightarrow \mathcal{L}(X \times K) \times L \rightarrow \mathcal{L}((X \times K) \times L)) .
$$

Again, one shows the result holds for all $E_{s}$. The limit ordinal case follows from the successor ordinal case, which in turn follows by induction and the fact that, for sets, the push-out of

$$
B \times K \stackrel{i \times K}{\longleftarrow} A \times K \stackrel{j \times K}{\longrightarrow} C \times K
$$

is isomorphic to $\left(B \cup_{A} C\right) \times K$. Note as well that the $\mathrm{Ex}^{\infty}$ functor is continuous in the same sense.

Suppose now that $f: A \rightarrow B$ is a cofibration of simplicial presheaves on a small Grothendieck site $\mathcal{C}$, and say that a simplicial presheaf $Z$ on $\mathcal{C}$ is $f$-local if $Z$ is globally fibrant and if the induced fibration

$$
f^{*}: \operatorname{hom}(B, Z) \rightarrow \operatorname{hom}(A, Z)
$$

is a weak equivalence.

We construct a functor $\mathcal{L}=\mathcal{L}_{f}: \mathbf{S} \operatorname{Pre}(\mathcal{C}) \rightarrow \mathbf{S} \operatorname{Pre}(\mathcal{C})$ by starting with an infinite cardinal $\alpha$ which is an upper bound for the cardinality of the set of morphisms of $\mathcal{C}$ and that of all sets of sections of $B$. The method of constructing the functor $\mathcal{L}$ is analogous to the simplicial set construction, starting with the set $\mathbf{C}$ of cofibrations having elements

$$
A \times L_{U} \Delta^{n} \cup_{A \times Y} B \times Y \hookrightarrow B \times L_{U} \Delta^{n},
$$

indexed over the set of all cofibrations $Y \subset L_{U} \Delta^{n}$.

Observe, however, that the construction of $\mathcal{L}$ for simplicial sets depends on the existence of a continuous functorial fibrant model construction. For simplicial presheaves, we require a continuous functorial globally fibrant model $j_{X}: X \rightarrow G X$ in order to carry out the analogous argument. The $\mathrm{Ex}^{\infty}$ functor does not produce globally fibrant models for simplicial presheaves, so we have to do something more interesting:

Lemma 4.1. Suppose that the infinite cardinal $\alpha$ is an upper bound for the cardinality of the set of morphisms of a small Grothendieck site $\mathcal{C}$. Then there is a functorial natural map $j_{X}: X \rightarrow G X$ such that the map $j_{X}$ is a trivial cofibration, $G X$ is globally fibrant, and the following properties hold:

G1: $G$ preserves weak equivalences. 
G2: $G$ preserves cofibrations.

G3: Let $\beta$ be any cardinal with $\beta \geq \alpha$. Let $\left\{X_{j}\right\}$ be the filtered system of sub-objects of $X$ which are $\beta$-bounded. Then the map

$$
\underset{j}{\lim } G X_{j} \rightarrow G X
$$

is an isomorphism.

G4: Let $\gamma$ be an ordinal number of cardinality strictly greater than $2^{\alpha}$. Let $X: \gamma \rightarrow \mathbf{S} \operatorname{Pre}(\mathcal{C})$ be a diagram of cofibrations so that for all limit ordinals $s<\gamma$ the induced map

$$
\underset{t<s}{\lim _{t<}} X(t) \rightarrow X(s)
$$

is an isomorphism. Then $\lim _{t<\gamma} G X(t) \cong G\left(\lim _{t<\gamma} X(t)\right)$.

G5: Suppose that $\lambda=2^{\kappa}$, where $\kappa$ is the defining cardinal for the functor $G$. If $X$ is $\lambda$-bounded, then $G X$ is $\lambda$-bounded.

G6: Let and $Y, Z$ be two subobjects of $X$. Then

$$
G(Y) \cap G(Z)=G(Y \cap Z)
$$

in $G(X)$.

G7: The functor $G$ extends to a natural morphism of simplicial sets

$$
G: \operatorname{hom}(X, Y) \longrightarrow \operatorname{hom}(G X, G Y)
$$

compatible with composition.

Proof: As before, the defining cardinal $\kappa$ for the functor $G$ is a cardinal $\kappa>$ $2^{\alpha}$. We begin by writing $C_{i} \rightarrow D_{i}$ for the set of trivial cofibrations $Y \subset L_{U} \Delta^{n}$, where $U$ varies through the set of objects of $\mathcal{C}$. Note that there are at most $2^{\alpha}$ trivial cofibrations $Y \subset L_{U} \Delta^{n}$, and that each such $Y$ is $\alpha$-bounded. Then $G X=$ $\lim _{s<\kappa} G_{s} X$ where $G_{s} X=\lim _{t<s} G_{t} X$ at limit ordinals $t<\kappa$, and $G_{s+1} X$ is constructed from $G_{s} X$ by requiring that the following diagram is a pushout

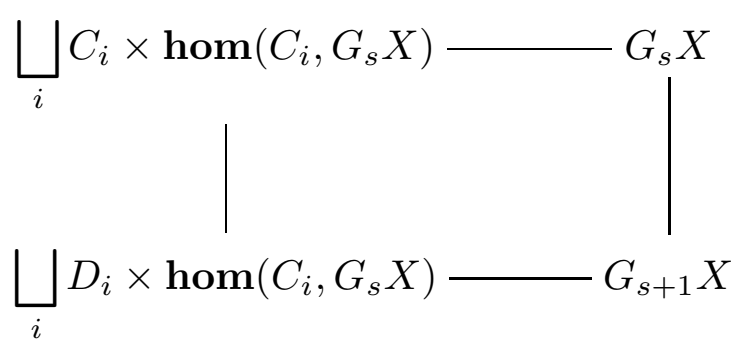


Each of the maps

$$
\bigsqcup_{i} C_{i} \times \operatorname{hom}\left(C_{i}, G_{s} X\right) \rightarrow \bigsqcup_{i} D_{i} \times \operatorname{hom}\left(C_{i}, G_{s} X\right)
$$

is a trivial cofibration of simplicial presheaves, so that the induced canonical map $j_{X}: X \rightarrow G X$ is a trivial cofibration. $G X$ is globally fibrant, since any map $C_{i} \rightarrow G X$ factors through some $G_{s} X$ with $s<\gamma$. In particular, G1 is a triviality. The statements $\mathbf{G 2}-\mathbf{G} 7$ are proved by analogy with L2 - L7 above.

To construct $\mathcal{L} X$ for a simplicial presheaf $X$, take $\kappa$ to be a cardinal greater than $2^{\alpha}$, and let $E_{0} X=G X$. Define $E_{s} X=G\left(\lim _{t<s} E_{t} X\right)$ at limit ordinals $s<\kappa$, where $G$ is the globally fibrant model construction of Lemma 4.1. At successor ordinals, $E_{s+1} X=G\left(\bar{E}_{s+1} X\right)$, where $\bar{E}_{s+1}(X)$ is defined by the pushout

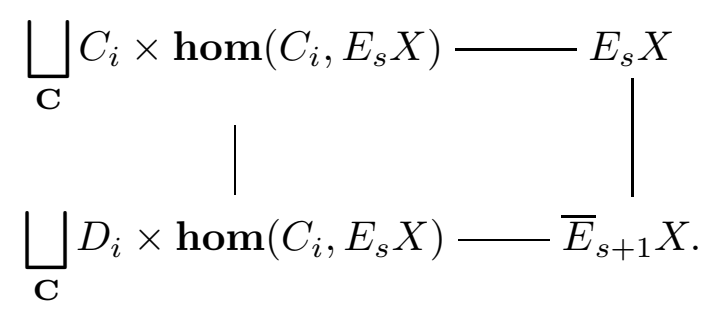

The notation $C_{i} \rightarrow D_{i}$ refers to all simplicial presheaf morphisms

$$
A \times L_{U} \Delta^{n} \cup_{A \times Y} B \times Y \hookrightarrow B \times L_{U} \Delta^{n} .
$$

Finally,

$$
\mathcal{L} X=\underset{s<\kappa}{\lim _{s}} E_{s} X
$$

Note that $\mathcal{L} X$ is globally fibrant: any map $Y \rightarrow \mathcal{L} X$ must factor through some $E_{s} X$ since $\kappa>2^{\alpha}$, and all $E_{s} X$ are globally fibrant by construction. A similar argument shows that $\mathcal{L} X$ is $f$-local. In the presence of Lemma 4.1, arguments for the simplicial presheaf analogues of statements $\mathbf{L} \mathbf{1}-\mathbf{L} \mathbf{7}$ go through just as before. In particular, we have proved

Theorem 4.2. Let $f: A \rightarrow B$ be a cofibration in $\mathbf{S}$ Pre $(\mathcal{C})$, and suppose that $\alpha$ is an infinite cardinal which is an upper bound for the cardinalities of both $B$ and the set of morphisms of $\mathcal{C}$. Then there is a functor $\mathcal{L}=\mathcal{L}_{f}: \mathbf{S P r e}(\mathcal{C}) \rightarrow \mathbf{S} \operatorname{Pre}(\mathcal{C})$ and a natural map $\eta_{X}: X \rightarrow \mathcal{L}(X)$ so that $\mathcal{L}(X)$ is $f$-local and $\eta_{X}$ is a cofibration which induces weak equivalences

$$
\eta_{X}^{*}: \operatorname{hom}(\mathcal{L}(X), Z) \rightarrow \operatorname{hom}(X, Z)
$$

for all $f$-local simplicial presheaves $Z$. 
As above, this yields a localization on the homotopy category.

We now use Theorem 1.1 to produce the $f$-local category structure on $\mathbf{S} \operatorname{Pre}(\mathcal{C})$. The following result allows us to identify the class $\mathbf{E}$ of $f$-local equivalences.

Lemma 4.3. Let $g: X \rightarrow Y$ be a morphism of simplicial pre-sheaves in $\mathbf{S} \operatorname{Pre}(\mathcal{C})$. The following two statements are equivalent.

1) $\mathcal{L} g: \mathcal{L} X \rightarrow \mathcal{L} Y$ is a weak equivalence

2) $g^{*}: \operatorname{hom}(Y, Z) \rightarrow \operatorname{hom}(X, Z)$ is a weak equivalence for all $f$-local objects $Z$ in $\mathbf{S} \operatorname{Pre}(\mathcal{C})$.

3) $[Y, Z] \rightarrow[X, Z]$ is an isomorphism for all $f$-local $Z$ in $\mathbf{S} \operatorname{Pre}(\mathcal{C})$.

Proof: Note that if $C \rightarrow D$ is any $f$-cofibration and $Z$ is $f$-local, then

$$
\operatorname{hom}(D, Z) \rightarrow \operatorname{hom}(C, Z)
$$

is a weak equivalence. Now examine the following diagram.

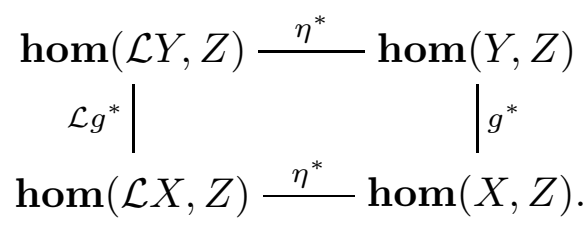

Since $\eta$ is an $f$-local equivalence, the horizontal maps are weak equivalences. Thus if $\mathcal{L} g: \mathcal{L} X \rightarrow \mathcal{L} Y$ is a weak equivalence, so is $g^{*}$. Thus (1) implies (2). We have that (2) implies (3) because any $f$-local object is globally fibrant. For (3) implies (1) note that for all $X$ and all $f$-local $Z$,

$$
[X, Z] \simeq[\mathcal{L} X, Z]
$$

since $\mathcal{L}$ induces the localization functor $L_{f}$ on the homotopy category. Thus (3) says $[\mathcal{L} Y, Z] \rightarrow[\mathcal{L} X, Z]$ is an isomorphism for all $f$-local $Z$. Since $\mathcal{L} X$ and $\mathcal{L} Y$ are $f$-local, this implies that $\mathcal{L} g: \mathcal{L} X \rightarrow \mathcal{L} Y$ is an isomorphism in the homotopy category; $\mathcal{L} g$ is therefore a weak equivalence (see Lemma II.4.1 of [9]).

We now define $\mathbf{E}$, the class of $f$-local equivalences, by the three equivalent conditions of Lemma 4.3. We next want to show $\mathbf{E}$ satisfies the seven axioms required by Theorem 1.1. Axioms E1-E3 are obvious, while Lemmas 4.4 and 4.5 handle axioms $\mathbf{E} 4-\mathbf{E} 6$ and $\mathbf{E 7}$, respectively.

LEMma 4.4. The class of $f$-local trivial cofibrations is closed under cobase change, colimits over ordinal numbers, and coproducts. 
Proof: Use (2) of Lemma 4.3, and the fact that trivial fibrations in simplicial sets are closed under base change, limits over ordinal numbers, and products.

LEMma 4.5. Let $\lambda=2^{\kappa}$, where $\kappa$ is the defining cardinal for the functor $\mathcal{L}$ (and $\kappa>$ $\left.2^{\alpha}\right)$. Then the class of $f$-local trivial cofibrations satisfies the bounded cofibration condition for the cardinal $\lambda$.

Proof: Let $X \rightarrow Y$ be an $f$-local equivalence and a cofibration, and let $A \subseteq Y$ be a $\lambda$-bounded sub-object. We inductively define a chain of $\lambda$-bounded sub-objects $A=A_{0} \subseteq A_{1} \subseteq A_{2} \subseteq \cdots \subseteq Y$ over $\lambda$, and a chain of sub-objects

$$
\mathcal{L}(A)=\mathcal{L}\left(A_{0}\right) \subseteq X_{1} \subseteq \mathcal{L}\left(A_{1}\right) \subseteq X_{2} \subseteq \mathcal{L}\left(A_{2}\right) \subseteq \cdots \mathcal{L}(Y),
$$

also over $\lambda$, with the property that

$$
\mathcal{L}(X) \cap X_{s} \rightarrow X_{s}
$$

is a weak equivalence. Then we set $B=\lim _{s<\kappa} A_{s}$ and, by L6,

$$
\begin{aligned}
\mathcal{L}(X \cap B)= & \mathcal{L}(X) \cap \mathcal{L}(B)=\underset{s<\kappa}{\lim _{\longrightarrow}} \mathcal{L}(X) \cap X_{s} \\
& \rightarrow \underset{s<\kappa}{\lim _{\vec{s}}} X_{s} \cong \mathcal{L}(B)
\end{aligned}
$$

is a weak equivalence as required.

The $A_{s}$ and $X_{s}$ are defined recursively. Suppose $s+1$ is a successor ordinal and $A_{s}$ has been defined. Then, since $A_{s}$ is $\lambda$-bounded, $\mathcal{L} A_{s}$ is $\lambda$-bounded by L5. Hence there is a $\lambda$-bounded sub-object $X_{s+1} \subseteq \mathcal{L}(Y)$ so that $\mathcal{L}\left(A_{s}\right) \subseteq X_{s+1}$ and $\mathcal{L}(X) \cap X_{s+1} \rightarrow X_{s+1}$ is a weak equivalence (see the proof of Theorem 2.7 with $f$ the identity functor, or argue directly). Since $\mathcal{L}(Y)=\lim _{\mathfrak{j}} \mathcal{L}\left(Y_{j}\right)$ where $Y_{j} \subseteq Y$ runs over the $\lambda$-bounded sub-objects of $Y$, there is a $\lambda$-bounded sub-object $A_{s+1}^{\prime}$ so that $X_{s+1} \subseteq \mathcal{L}\left(A_{s+1}^{\prime}\right)$. Let $A_{s+1}=A_{s} \cup A_{s+1}^{\prime}$. Finally, suppose $s$ is a limit ordinal. Then set $X_{s}=\lim _{t<s} \mathcal{L}\left(A_{t}\right) \cong \lim _{t<s} X_{t}$. The object $X_{s}$ is $\lambda$-bounded and $\mathcal{L}(X) \cap X_{s} \rightarrow X_{s}$ is a weak equivalence. Choose $A_{s}^{\prime} \subseteq Y$ so that $A_{s}^{\prime}$ is $\lambda$-bounded and $X_{s} \subseteq \mathcal{L}\left(A_{s}^{\prime}\right)$ and set $A_{s}=\lim _{t<s} A_{t} \cup A_{s}^{\prime}$.

Theorem 4.6. The category $\mathbf{S} \operatorname{Pre}(\mathcal{C})$ acquires the structure of a simplicial model category with ordinary simplicial presheaf cofibrations and $f$-local equivalences.

Proof: This follows from Theorem 1.1, once we verify condition (2) for that result. We will see in the next lemma that if $Z \in \mathbf{S} \operatorname{Pre}(\mathcal{C})$ is $f$-local and $K \in \mathbf{S}$, then $X^{K}$ is $f$-local. That said, let $K \rightarrow L$ be a cofibration in $\mathbf{S}$ and $C \rightarrow D$ a cofibration in $\mathbf{S} \operatorname{Pre}(C)$. Consider the cofibration in $\mathbf{S} \operatorname{Pre}(C)$

$$
D \times K \cup_{C \times K} C \times L \rightarrow D \times L .
$$


Let $Z$ be $f$-local and apply $\operatorname{hom}(\cdot, Z)$. Then there is an augmented pull-back square in $\mathbf{S}$

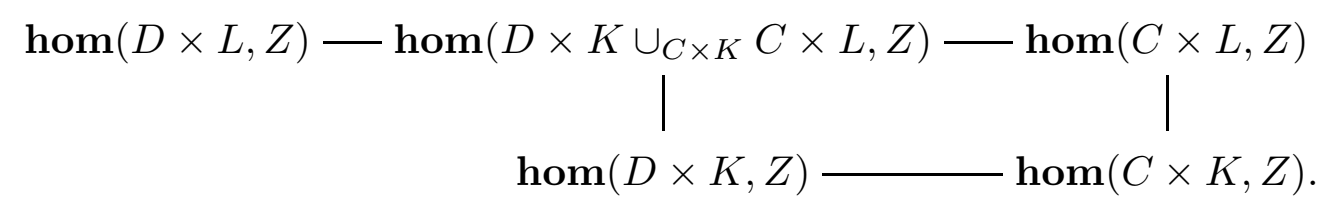

Now, there is a natural isomorphism of this diagram to

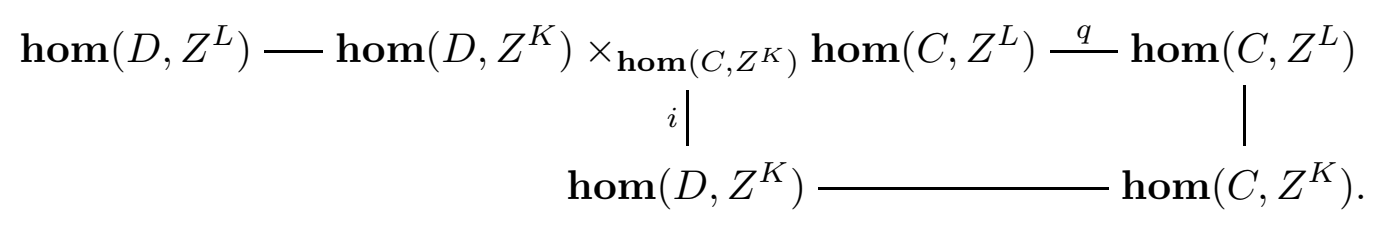

Now suppose $C \rightarrow D$ is an $f$-local equivalence. Then by Lemma 4.3.2,

$$
\operatorname{hom}\left(D, Z^{K}\right) \rightarrow \operatorname{hom}\left(C, Z^{K}\right)
$$

is trivial fibration; therefore $q$ is a trivial fibration. Since

$$
\operatorname{hom}\left(D, Z^{L}\right) \rightarrow \operatorname{hom}\left(C, Z^{L}\right)
$$

is a weak equivalence, (4.7) is an $f$-local equivalence. Similarly, if $K \rightarrow L$ is a weak equivalence, $\operatorname{hom}\left(C, Z^{L}\right) \rightarrow \operatorname{hom}\left(C, Z^{K}\right)$ is a weak-equivalence, since $Z$ is fibrant. Then $i$ is a weak equivalence and the result follows.

This leaves the following technical result.

Lemma 4.8. If $Z \in \mathbf{S} \operatorname{Pre}(\mathcal{C})$ is $f$-local and $K \in \mathbf{S}$, then $Z^{K}$ is $f$-local.

Proof: We need $\operatorname{hom}\left(B, Z^{K}\right) \rightarrow \operatorname{hom}\left(A, Z^{K}\right)$ to be a weak equivalence. But this fibration of simplicial sets is isomorphic to

$$
\operatorname{hom}_{\mathbf{S}}(K, \operatorname{hom}(B, Z)) \rightarrow \operatorname{hom}_{\mathbf{S}}(K, \operatorname{hom}(A, Z)),
$$

and $\operatorname{hom}(B, Z) \rightarrow \operatorname{hom}(A, Z)$ is a trivial fibration by hypothesis.

One final remark. While the fibrations in the $f$-local model category structure of $\mathbf{S} \operatorname{Pre}(\mathcal{C})$ are cloaked in mystery, we do have the following congruence.

Proposition 4.9. An object $X \in \mathbf{S} \operatorname{Pre}(\mathcal{C})$ is fibrant in the $f$-local model category structure if and only if it is $f$-local. 
Proof: First suppose $X$ is fibrant. Note that $f: A \rightarrow B$ is an $f$-local equivalence by Lemma 4.3.2. Thus $\operatorname{hom}(B, X) \rightarrow \operatorname{hom}(A, X)$ is a trivial fibration; hence $X$ is $f$-local.

Now suppose $X$ is $f$-local. Consider a lifting problem

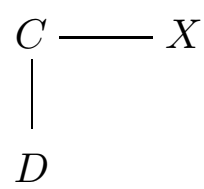

where $C \rightarrow D$ is a $f$-local trivial cofibration. Since $X$ is $f$-local there is a map $\mathcal{L}(C) \rightarrow X$ making the following diagram commute

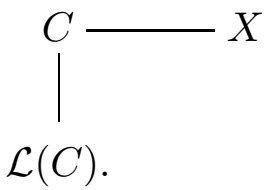

Since $\mathcal{L}(C) \rightarrow \mathcal{L}(D)$ is a trivial cofibration, there is a map $\mathcal{L}(D) \rightarrow X$ making

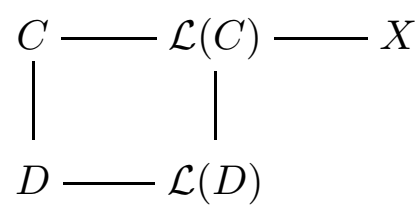

commute. This solves the original lifting problem.

REMARK 4.10 . Let $k$ be a field, and suppose that $\left(\left.S c h\right|_{k}\right)_{N i s}^{\kappa}$ is a small model for the big Nisnevich site on $k$, in the sense of Section 6.5 of [14]. The objects of this category consist of all schemes which are locally of finite type over $k$ and have cardinality of their underlying point sets and all rings of sections bounded above by $\kappa$. Here, $\kappa$ is just some infinite cardinal with $\kappa>|k|$.

The collection of all projections

$$
p r_{X}: X \times \mathbb{A}^{1} \rightarrow X
$$

in $\left(\left.S c h\right|_{k}\right)_{N i s}^{\kappa}$ forms a set, and represents a collection of presheaf maps and hence maps of (constant) simplicial presheaves. Each of the maps $p r_{X}$ can be replaced 
by a cofibration $f_{X}$ of simplicial presheaves up to weak equivalence, since there is a factorization

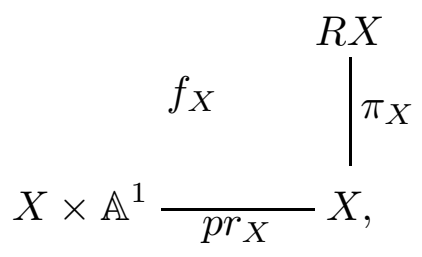

of $p r_{X}$ with $\pi_{X}$ a global fibration and a local weak equivalence and $f_{X}$ a cofibration. Let $f$ be the disjoint union of the maps $f_{X}$ :

$$
f=\bigsqcup_{X} f_{X}: \bigsqcup_{X} X \times \mathbb{A}^{1} \rightarrow \bigsqcup_{X} R X
$$

The Morel-Voevodsky $\mathbb{A}^{1}$-local theory [17] is the closed model structure on the category of simplicial presheaves on $\left(\left.S c h\right|_{k}\right)_{N i s}^{\kappa}$ which arises from Theorem 4.6 by localizing at the cofibration $f=\sqcup f_{X}$.

Note that we could equally well have started with a collection of sections $X \rightarrow$ $X \times \mathbb{A}^{1}$ of the corresponding projections. These have the advantage of being cofibrations already.

\section{BIBLIOGRAPHY}

[1]. M. Artin, A. Grothendieck and J-L. Verdier, SGA4, Théorie des Topos et Cohomologie Étale des Schémas, Springer Lecture Notes in Math. 269, Springer-Verlag, Berlin-Heidelberg-New York, 1972.

[2]. A.K. Bousfield, The localization of spaces with respect to homology, Topology 14 (1975), $133-150$.

[3]. A.K. Bousfield, The localization of spectra with respect to homology, Topology 18 (1979), $257-281$.

[4]. A.K. Bousfield, Localization and periodicity in unstable homotopy theory, Journal of the AMS 7 (1994), 31-874.

[5]. A.K. Bousfield and E.M. Friedlander, Homotopy theory of $\Gamma$-spaces, spectra, and bisimplicial sets, Springer Lecture Notes in Math. 658 (1978), 80-150.

[6]. E. Dror-Farjoun, The localization with respect to a map and $v_{1}$ periodicity, in Algebraic Topology: Homotopy and Group Cohomology, J. Aguadé, M. Castellet, and F.R. Cohen, eds., Springer Lecture Notes in Math. 1509 (1992).

[7]. E. Dror-Farjoun, Cellular Spaces, Null Spaces, and Homotopy Localization, Springer Lecture Notes in Math. 1622 (1996).

[8]. H. Gillet and C. Soulé, Filtrations on Higher Algebraic K-Theory, Preprint (1983).

[9]. P.G. Goerss and J.F. Jardine, Simplicial Homotopy Theory, Preprint (1996), http://www.math.uwo.ca/ jardine/papers/simp-sets.

[10]. P. Hirschhorn, Localization, Cellularization, and Homotopy Colimits, Preprint (1996)..

[11]. J.F. Jardine, Simplicial objects in a Grothendieck topos, Contemporary Math. 55 (1986), 193-239.

[12]. J.F. Jardine, Simplicial presheaves, J. Pure Applied Algebra 47 (1987), 35-87. 
[13]. J.F. Jardine, Stable homotopy theory of simplicial presheaves, Can. J. Math. 39 (1987), $733-747$.

[14]. J.F. Jardine, Generalized Etale Cohomology Theories, Progress in Math., Vol. 146, Birkhäuser, Basel-Boston-Berlin, 1997.

[15]. J.F. Jardine, Boolean localization, in practice, Doc. Math. 1 (1996), 245-275.

[16]. S. Mac Lane and I.Moerdijk, Sheaves in Geometry and Logic: A First Introduction to Topos Theory, Springer-Verlag, Berlin Heidelberg New York, 1992.

[17]. V. Voevodsky, The Milnor Conjecture, Preprint, (1996).

Mathematics Department, University of Washington, Seattle, WA 98195, USA.

E-mail: pgoerss@math.washington.edu

Mathematics Department, University of Western Ontario, London, Ont. N6A 5B7, Canada. E-mail: jardine@uwo.ca 